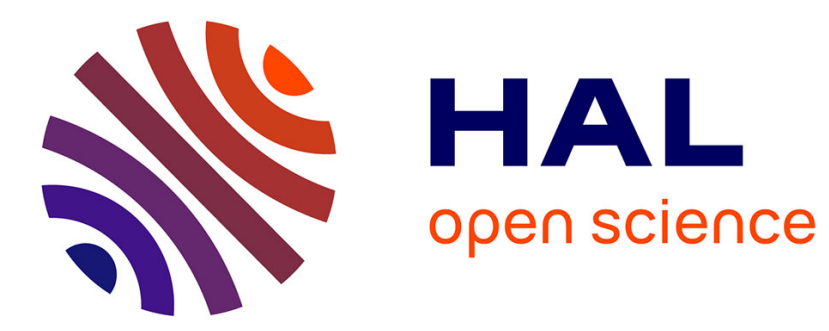

\title{
IX. Études cam. II. L'introduction de l'Islam au Campa Pierre-Yves Manguin
}

\section{To cite this version:}

Pierre-Yves Manguin. IX. Études cam. II. L'introduction de l'Islam au Campa. Bulletin de l'Ecole française d'Extrême-Orient, 1979, 66 (1), pp.255-287. 10.3406/befeo.1979.4016 . halshs-02508910

\section{HAL Id: halshs-02508910 https://shs.hal.science/halshs-02508910}

Submitted on 16 Mar 2020

HAL is a multi-disciplinary open access archive for the deposit and dissemination of scientific research documents, whether they are published or not. The documents may come from teaching and research institutions in France or abroad, or from public or private research centers.
L'archive ouverte pluridisciplinaire HAL, est destinée au dépôt et à la diffusion de documents scientifiques de niveau recherche, publiés ou non, émanant des établissements d'enseignement et de recherche français ou étrangers, des laboratoires publics ou privés. 


\section{IX. Études cam. II. L'introduction de I'Islam au Campa}

Pierre-Yves Manguin

\section{Citer ce document / Cite this document :}

Manguin Pierre-Yves. IX. Études cam. II. L'introduction de I'Islam au Campa. In: Bulletin de l'Ecole française d'ExtrêmeOrient. Tome 66, 1979. pp. 255-287;

doi : https://doi.org/10.3406/befeo.1979.4016

https://www.persee.fr/doc/befeo_0336-1519_1979_num_66_1_4016

Fichier pdf généré le 08/11/2019 


\title{
ETUDES CAM
}

\author{
Il. L'INTRODUGTION DE L'ISLAM AU GAMPĀ *
}

PAR

Pierre-Yves MANGUIN

En 1927, alors que les études sur le Campā battaient leur plein sous l'égide de l'École française d'Extrême-Orient, A. Cabaton avait avancé deux hypothèses pouvant expliquer l'introduction de l'Islam en Indochine et plus particulièrement en pays cam : il pouvait y avoir été amené par des marchands arabes, persans ou indiens, du $\mathrm{x}^{\mathrm{e}} \mathrm{au} \mathrm{xIv}^{\mathrm{e}}$ siècles, ou bien il s'y était implanté plus tard "grâce à une immigration malaise "1. Cabaton, manquant alors d'éléments, ne se prononçait franchement pour aucune de ces deux hypothèses. Un demi-siècle plus tard, après une longue interruption des études cam, de nouveaux éléments peuvent être amenés à ce dossier, qui concernent pour l'essentiel la période postérieure à la chute de la capitale cam Vijaya en $1471^{2}$. La présentation de ces quelques résultats m'a paru être une bonne occasion de rassembler, de dresser en quelque sorte le catalogue des

(*) Cet article doit beaucoup aux travaux, aux suggestions et aux informations fournies par les membres du groupe de recherches cam de l'École pratique des Hautes Études (IVe section), MM. P.-B. Lafont, Nara vija, Pó Dharma, G. Moussay et C. Jacques. Il a également bénéficié des conseils de M. D. Lombard. Qu'ils en soient tous ici remerciés. La translittération utilisée est celle qui a été mise au point par ce même groupe de recherches ("Essai de translittération raisonnée du Cam ", BEFEO, LXIV, 1977, pp. 243-255). Voir aussi le catalogue des manuscrits cam récemment publié (Lafont, Pó Dharma et Nara Vija, 1977).

(1) Encyclopédie de l'Islam, Leyde, 1927, s.v. Indochine, p. 538. Cabaton avait déjà émis cette hypothèse, en des termes identiques, dans ses "Notes sur l'Islam dans l'Indochine française "(Cabaton, 1906, p. 30). Mus (1931) n'ajoutait rien de mieux en ce qui concerne l'Islam cam.

(2) En ce qui concerne plus particulièrement les sources cam, la thèse récente de Pó Dharma ( 1978 ; inédite) apporte d'intéressants éléments et une vue nouvelle sur les textes qu'Aymonier qualifiait de "chroniques cam". Se basant sur un plus grand nombre de textes (près de 60) et sur une meilleure connaissance de la langue cam, ce travail remplace avantageusement les traductions peu fiables effectuées, par Aymonier surtout, alors que les études cam en étaient à leurs débuts. 
éléments très épars de cette étude, non pour résoudre ici tous les problèmes qui se posent - et qui demandent des compétences diverses -, mais bien pour les poser et les réexaminer à la lumière des recherches plus récentes sur l'Islam en Asie du Sud-Est et plus particulièrement dans le monde nousantarien.

Les études d'histoire cam ont en effet souffert d'avoir été longtemps menées comme une sorte d'annexe des études viêtnamiennes, ou, au mieux, "indochinoises ". Le Campā, de langue et de culture austronésiennes, appartient au monde nousantarien ${ }^{1}$. De plus, et de même que l'Insulinde, le Campā ne peut être isolé des réseaux maritimes tissés dans le Sud-Est asiatique et dans l'Asie toute entière. Son étude doit s'inscrire dans une problématique plus vaste.

Le déchiffrement en 1922 par $P$. Ravaisse de deux inscriptions arabes constitue certainement la preuve la plus tangible de l'existence d'une communauté musulmane au Campā au XI ${ }^{\mathrm{e}}$ siècle, si, bien entendu, on accepte leur localisation sur les côtes de ce pays, très vraisemblable mais non pas certaine ${ }^{2}$. La première stèle, datée du 21 novembre 1039, marquait la tombe d'un certain Abū Kāmil "le garde-chemins". L'écriture en est «du pur coufique fāṭimite, conçu et exécuté d'après les traditions classiques». La deuxième, en coufique très rudimentaire, est très endommagée, mais Ravaisse put tout de même en déduire qu'il s'agissait "d'un acte public destiné à aviser les membres d'une colonie d'Arabes, de Persans et de Turcs, de la façon dont ils doivent en user avec les gens du pays dans leurs transactions, leurs opérations de change et le paiement de leurs contributions ". Sa date manque, mais elle paraît bien être contemporaine de la précédente.

La présence de ces deux stèles n'indique rien d'autre que l'existence au $\mathrm{XI}^{\mathrm{e}}$ siècle d'une communauté de marchands musulmans, étrangers

(1) Louis-Charles Damais a été le premier à inclure des conférences sur le Campà à son séminaire de "Langues et Civilisations indonésiennes" à l'École pratique des Hautes Études, à partir de 1964 et jusqu'à sa mort. Mais ses recherches n'ont donné lieu à aucune publication (on se reportera aux résumés de ses conférences publiés dans l'Annuaire de l'EPHE, $I V$ e section, aux années correspondantes).

(2) Ravaisse, 1922. Il faut rappeler ici les conditions pour le moins douteuses dans lesquelles ces inscriptions ont été révélées : Ravaisse reçut les deux estampages par deux personnes interposées, une vingtaine d'années après que les stèles, qui n'ont jamais été retrouvées, aient été découvertes "sur un point non éloigné de la côte annamite, par un officier de la marine française dont on ne connaît plus le nom ". Il serait bon que tout raisonnement s'appuyant sur l'existence au Campā de ces stèles tienne compte du doute qui pèse sur l'authenticité de ces témoignages. Ravaisse, s'appuyant sur des données largement dépassées aujourd'hui (les vallées de Phan-rang et Phan-ri, "lieu d'origine des Cam "), pensait, sous toutes réserves, que ces stèles pouvaient vraisemblablement provenir du Pāṇụuranga. Fatimi (1963, pp. 43, 59), qui leur fait une place importante dans son étude, les qualifie tout bonnement "d'inscriptions de Phan-rang". Rien ne prouve en fait que ces estampages aient été faits au Pāndurañăga. La " côte annamite "s'étend jusqu'au Nord de Huê. Mais, dès l'an 1000, la capitale du Campā s'est repliée vers le sud à Vijaya, dans le Bình-dịnh. Ceci dit, le Pāndụangang ne peut être exclu d'office : il était assez important pour proclamer son indépendance dans la deuxième moilié du xi ${ }^{\text {e }}$ siècle (Maspero, 1928, p. 148 ; Boisselier, 1963, p. 312). 
au pays. Après avoir envisagé diverses autres hypothèses, Ravaisse n'a rien affirmé de plus dans son commentaire ${ }^{1}$. Quoi de plus normal que cette implantation lorsqu'on sait que des marchands musulmans résidaient alors en si grand nombre dans la Chine méridionale depuis le viII ${ }^{\mathbf{e}}$ siècle. Le comptoir du Campā n'est ainsi que l'un des maillons d'une longue chaîne reliant le Moyen-Orient à l'Afrique et à la Chine. La stèle funéraire de Lcran (datée de 1082) témoigne de même de l'existence d'une communauté musulmane à Java, près du port de Gresik (on remarquera que sous le règne d'Airlanga, c'est-à-dire à l'époque même où ont été rédigées les stèles du Campā, des épigraphes citent les Cam parmi ceux qui fréquentent le port de Gresik) ${ }^{2}$. Ces communautés sont "avant tout, il faut y insister, des supports du transit et du négoce nés des initiatives privées et des besoins du grand commerce lointain " ${ }^{3}$. Le Campā n'était-il pas "borné par des mers où passent les navires de tous les pays " ${ }^{4}$ ? Ravaisse s'étonne même que ce pays n'ait pas connu l'implantation de colonies musulmanes étrangères plus conséquentes : mais si le Campā pouvait en raison de sa position exercer une forme de contrôle sur la route maritime transasiatique, il était économiquement peu important, un petit maillon de ce réseau, dans lequel il injectait quelques produits de luxe tirés de ses forêts. Rien n'y justifiait l'installation d'une colonie marchande étrangère aussi importante que celle de la Chine méridionale.

Les géographes arabes et persans mentionnent souvent les colonies musulmanes de la Chine méridionale, mais ils sont dans leur majorité très brefs lorsqu'ils parlent du Campā et se contentent de remarquer que c'est le pays qui produit le meilleur bois d'aloès. Il existe cependant un témoignage qui vient confirmer la présence de musulmans moyenorientaux dans ce pays. L'auteur en est al-Dimashki. Sa cosmographie, écrite vers 1325 , est confuse et souvent sujette à caution, mais elle contient néanmoins des indications originales, comme en témoigne la citation suivante ${ }^{5}$ :

«(...) le pays de Campā, avec la ville principale du même nom... peuplée de musulmans, de chrétiens et d'idolâtres. La religion musulmane y est venue du temps de 'Othmän (...) et les Alides, expulsés par les Oméyyades et par Hadjdjādj s'y sont réfugiés (...) $1^{6}$.

(1) Fatimi, 1963, pp. 46-47, dans son commentaire de cette inscription, parvient a préciser qu'elle a été rédigée par des Shi'ites, en se fondant sur les articles de foi et les attributs de Dieu donnés dans l'inscription. Ceci est confirmé par ce qu'on sait par ailleurs de l'origine des colonies musulmanes moyen-orientales en Extrême-Orient (cf. infra).

(2) Ravaisse, 1925 ; Damais, 1968, pp. 570-571. Pour les inscriptions du temps d'Airlanga, cf. Krom, 1931, p. 264 .

(3) Miquel, 1975, pp. 516-522. Voir aussi Arnold, 1935, p. 263.

(4) Passage du Linyi ji, écrit au ve siècle (trad. par Aurousseau, $B E F E O, X I V / 9,1914$, p. 13). Sur la position stratégique du Campā sur la route maritime transasiatique, cf. Manguin, 1973.

(5) Ferrand, 1914, 11, pp. 363-393 (citation p. 391).

(6) 'Othmān, le troisième calife, est mort en 656. Les Ummayades ont pris le pouvoir entre cette date et 661 (assassinat d'Alì). Madjajädj, gouverneur de l'Irak en 694, les a défendus iusqu'à sa mort en 714 . Il semble que ces dates, qui marquent bien les débuts de la fuite des 
Dans les années 1320, c'est-à-dire cinq ou six siècles après les événements relatés, il ne faut certainement voir là que le souvenir de l'implantation d'un comptoir alide au Campā, sans prendre à la lettre le "peuplement" de la capitale cam". On rappellera que l'inscription de la tombe d'Abū Kamil "le garde-chemins ", d'après Fatimi, semble bien relever de l'école shì'ite.

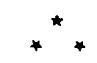

Les sources Song fournissent une deuxième série d'informations ayant trait à l'Islam et au Campā. Elle concerne les personnages qui, en Asie du Sud-Est et dans les ports de la Chine méridionale, portent le nom de famille que les Chinois transcrivent en général par pu 蒲 ${ }^{2}$. A la suite de Hirth, Rockhill, Kuwabara et Lo Xiang-lin ${ }^{3}$, il a généralement été accepté que ce $p u$ représentait l'arabe $A b \bar{u}$ et que, par conséquent, tous ceux qui portaient ce patronyme étaient soit arabes, soit simplement musulmans. G. Ferrand a pour sa part dénoncé cette théorie, qu'il qualifie d'idola libri, et a proposé de voir dans pu une transcription du titre nousantarien $p u / m p u / p \check{o}^{4}$. W. Franke et Ch'en T'ieh-fan, après avoir noté que le patronyme $P u$ est attesté en Chine dès les Han et qu'il y est par ailleurs utilisé à divers titres, ont émis l'hypothèse que tous ceux qui portent un tel nom ne sont pas nécessaire-

\footnotetext{
Alides vers l'Est, soient trop hautes pour l'installation en Inde et en Extrême-Orient de véritables comptoirs de marchands musulmans arabo-persans. La fondation du centre urbain de Bagdad en 762 - avec Bașra, son débouché vers l'Océan Indien - en transformant les conditions du marché, contribua au développement du commerce entre le Golfe Persique et l'Extrême-Orient et c'est d'alors que date la véritable expansion du commerce du monde islamique avec l'Extrême-Orient (M. Lombard, 1971, pp. 126-127, 221-223: Sauvaget, 1948, pp. XXXVI-XXXvir).

(1) La population chrétienne de la capitale n'est attestée nulle part ailleurs. Mais l'on sait que les Nestoriens, nombreux alors en Chine méridionale, fréquentaient aussi les routes maritimes de la Mer de Chine (Phillips, 1879-80). Voir aussi l'exemple donné par Abūlfidā d'un moine nestorien qui se rendit en Chine par mer et lui fournit des informations, précisément, sur le Campā (Reinaud, 1848, I, CDXvi).

(2) On laissera de côté la note de Huber où il voyait, dans une invocation cam pendant le sacrifice du buffle, transcrite dans le Song shi par a lo he qi ba, un très hypothétique Allähu akbar ( $q$ i ba pourrait aussi bien transcrire le cam lapaw, "bume "). Sur cette unique prémisse, Huber affirmait qu'il y avait des Cam convertis a l'Islam du temps des Song (note in : Durand, 1903 , p. 55).

(3) Hirth, 1896, p. 487 ; Hirth et Rockhill, 1911, p. 16, 60, 64, 280 ; Kuwabara, 19281935 ; Lo Xiang-lin, 1955, 1959 ; voir aussi Tasaka, 1952.

(4) Ferrand, 1922, p. 9, n. 2. Il n'envisage dans cette note que le cas précis de Srivijaya, dont le Zhufanchi dit succinctement que de nombreux habitants ont pour nom de famille $P u$. Mais il aurait dû considérer les nombreux exemples tirés des sources chinoises où il semble bien que l'on puisse voir des transcriptions de noms arabes : c'est le cas des envoyés du pays des Dashi (les Arabes). Sur des missions conduites par des $P u$ et venues d'autres pays de l'Asie du Sud-Est el du Moyen-Orient, cf. Hirth et Rockhill, 1911, pp. 52, 122-123, 157 ; Kuwabara, 1935, pp. 3, 18-20; Fatimi, 1963, p. 68. P. Pelliot, en commentant la valeur de transcription de $p u$, semble accepter la remarque de Ferrand (Pelliot, 1963, II, p. 389). Un troisième auteur a plus récemment traité de ce problème, mais je n'ai pu avoir accès à cet article (Kikuchi Saburo, 1969).
} 
ment d'origine arabe (ou musulmans de religion) ${ }^{1}$. En attendant que ce problème ait fait l'objet d'une étude plus approfondie de la part des sinologues, aucune réponse définitive ne peut être donnée en la matière.

Ce problème, qui concerne tant l'Asie du Sud-Est que la Chine méridionale, intéresse les études cam à plus d'un titre. Lorsque le roi cam renoue ses relations avec la Chine - qui commence à se réunifier - il $\mathrm{y}$ envoie en 951 une première ambassade; dans le courant des $\mathrm{x}^{\mathrm{e}}$ et $\mathrm{XI}^{\mathrm{e}}$ siècles, de nombreuses autres suivront. Parmi les ambassadeurs, quelques-uns ont pour premier élément du nom qui leur est attribué par les historiens chinois le caractère $p u$ 蒲. On a souvent cité à l'appui de la thèse $A b \bar{u}>P u$ le premier ambassadeur $P u H e$ San 蒲韵散 - qui se rendit en Ghine à plusieurs reprises entre 951 et 972 - en restituant son nom en $A b \bar{u}$ l-Ḩasan. Outre celui-ci, d'autres ambassadeurs ont des noms qui commencent par $p u$ (蒲 ou 莆), $b u$ (布) ou $b o(\text { 波 })^{2}$. On sait par ailleurs que les célèbres familles musulmanes $P \boldsymbol{P u}$ de Guangzhou (Canton) et de Quanzhou, d'origine arabe et qui étaient arrivées en Chine sous les Song, avaient auparavant séjourné au Campa $\bar{a}^{3}$.

Une autre série d'événements rapportés par ces mêmes sources chinoises écrites, complétées par certaines traditions orales, semble encore confirmer la présence de colons musulmans sur les côtes cam. A la suite de l'usurpation de la couronne cam par le viêtnamien Lưu Ký Tông, il se produisit à partir de 986 une émigration du Campā vers la Chine méridionale. Une centaine "d'étrangers du Campā 》占城夷人 arrivèrent cette année-là à Hainan, menés par un certain $P u L o E$ 蒲羅遏. Près de cinq cents autres demandèrent à Canton la protection de la Chine ; ils avaient à leur tête Li Nianq Binq 李娘并 en 987 et $H u X u a n$ 忽宣 en $988^{4}$. Si l'on admet, comme cela a été plusieurs fois proposé, la restitution de $H u X u a n$ en Husain ( $h u / \chi u \partial t$ rendant aisément le $h$ arabe), il s'agit encore de musulmans venus du Campa $\bar{a}^{5}$. Or, l'une des traditions de la minorité musulmane de Sanya shi, port du Sud de Hainan faisant face aux côtes indochinoises, veut que cette population ait préalablement séjourné au Campā $\bar{a}^{6}$. Ceci est confirmé par diverses

(1) Ch'en T'ieh-fan et Franke, 1973. L'inscription révélée dans cet article est celle de la tombe d'un "sieur $P u$ "; c'est la plus ancienne inscription chinoise datée de l'Asie du Sud-Est maritime.

(2) On trouvera la liste de ces ambassades rassemblée dans le Song shi (chap. 489, pp. 3b-11a de l'éd. Pona). Pour des références à des sources chinoises complémentaires, voir aussi Maspero, 1928, pp. 119-151. Mais on prendra soin de se reporter aux textes, car Maspero, en ces quelques pages, accumule un nombre considérable de mauvaises lectures, transcriptions et traductions ; cet auteur laisse par ailleurs complètement de côté le problème de la restitution du nom de ces ambassadeurs.

(3) Le membre le mieux connu de cette famille est Pu Shougeng; il remplissait à la fin des Song une haute charge : il était commissaire des douanes et passa au service des Yuan en 1270 avec toute sa flotte. Une autre famille $\mathrm{Pu}$, dont les membres remplirent aussi de hautes charges, dont celle de chef du quartier des étrangers, résidait à Canton. Mais contrairement à ce qu'afirme Kuwabara, ils ne sont pas de la même famille que le Pu Shougeng de Quanzhou (Kuwabara, 1928-35; Lo Xianglin, 1959).

(4) Song shi, 489, pp. 4b-5a ; Maspero, 1928, pp. 123-125.

(5) Kuwabara, 1935, p. 22 ; il propose aussi de restituer Pu Lo E en Abū Rao.

(6) Stübel, 1937, pp. 263-265, 315-317. Stübel écrit "Annam", mais il faut y voir le Campā. La date fournie par la tradition orale est par contre difficile à accepter : la migration 
sources écrites, dont la généalogie de la famille $\mathrm{Pu}$ de Sanya shi, qui précisent bien que ces "étrangers" du Sud de Hainan sont venus du Campa ${ }^{1}$. Une étude linguistique - malheureusement assez superficielle car effectuée sur le vocabulaire très succinct ramené par Stübel - a reconnu enfin des affinités certaines entre la langue de cette communauté musulmane et le cam (où, en tout état de cause, une langue austronésienne dans laquelle un substrat mon-khmer est apparent) ${ }^{2}$.

A ce stade du présent travail, il faut tout au moins noter qu'on ne peut ignorer la convergence de l'ensemble de ces sources chinoises et des sources arabes précédemment citées, qui établit sans conteste à partir du viı ${ }^{e}$ siècle des liens entre le Campā, les colonies de marchands musulmans de la Chine méridionale et les familles $P u$. Sans préjuger des résultats qui seront fournis par de nouvelles études sur l'Islam extrême-oriental et l'usage qui est fait par les sources chinoises du patronyme $P u$, l'hypothèse la plus vraisemblable consiste bien à réduire l'ensemble de ces témoignages à l'existence au Campā, à partir du $\mathrm{x}^{\mathrm{e}}$ siècle certainement et peut-être déjà à une plus haute époque, d'une communauté de marchands moyen-orientaux musulmans qui entretenaient des rapports étroits avec leurs coreligionnaires de la Chine méridionale. Qu'une telle colonie ait eu des contacts avec la population cam, c'est évident. Que des Cam se soient convertis à leur rencontre, qu'il y ait eu des mariages mixtes, c'est encore fort possible. Mais, comme dans le reste de l'Asie du Sud-Est, ces conversions n'ont alors vraisemblablement constitué que des phénomènes isolés, limités aux sphères commerçantes en relation avec les communautés étrangères. Ces musulmans étrangers (ou Cam convertis) pouvaient d'ailleurs être en contact direct avec le souverain hindouiste. On sait par Ibn Rusteh, qui écrivait vers 903 , qu'un négociant arabe avait séjourné deux ans à la cour du souverain $\mathrm{khmer}^{3}$. On pourrait ainsi fort bien penser que le roi cam, connaissant la position de force des colonies musulmanes en Chine, se soit fait représenter auprès de la cour impériale par des marchands arabes résidant au Campā, ayant d'utiles contacts en Chine et dont la langue, contrairement au cam, devait être facilement comprise

aurait eu lieu il y a qualre cents ans seulement. Savina $(1929, \mathrm{pp} .4,22)$ parle à tort de "malais venus s'échouer sur la còte Sud ".

(1) Kuwabara, 1935, pp. 21-22 et Lo Xianglin, 1959, pp. 165-226 (où est publiće la généalogie des Pu de Ilainan).

(2) Benedict, 1941. Mais une communauté d'orig̣ine moyen-orientale installée quelque temps au Campā aurait-clle abandonné sa langue maternelle pour adopter le Cam ? Si l'on en croit les stèles traduites par liavaisse, il semble bien que non. S'agit-il alors de Cam convertis? Mais Stübel, qui rapporte le souvenir qu'ils gardent de leur origine moyenorientale, a décelé chez eux des traits somatiques sémites. Peut-être est-on en présence de descendants de mariages entre arabes et cam? Il semble néanmoins qu'en ce qui concerne les populations de Hai-nan et de la Chine méridionale, le problème soit plus complexe encore et déborde largement le cadre de l'Islam. Le rôle joué aux Ier $^{\text {et }}$ I $^{\mathrm{e}}$ siecles dans la formation du Linyi primitif (futur Campā) par des "barbares " plus ou moins soumis à l'administration chinoise reste encore obscur, mais il est attesté sans équivoque. Les Li de Hai-nan ont à cet égard une place privilégiée (Stein, 1947, pp. 116, 130-145, 241 sq.). Les Tanka aussi sont dits venir du Linyi (Ho Kê-ên, 1959-60, p. 3, 1967, p. 123 ; Stein, 1947, pp. 307-309).

(3) Ferrand, 1914, I, p. 69. 
dans les milieux chinois liés au commerce extérieur. On connaît d'autres exemples d'ambassadeurs choisis par les souverains asiatiques parmi les marchands des colonies étrangères ${ }^{1}$. Mais si ces étrangers musulmans ont pu de ce fait exercer au Campā un certain pouvoir en matière commerciale et diplomatique, il est peu vraisemblable qu'ils aient dès lors influencé la culture du peuple cam $^{2}$.

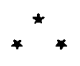

Dans la littérature et les traditions orales de Java figure souvent un pays nommé Cĕmpa qui est en général associé à l'introduction de l'Islam dans l'île, et à la chute de Majapahit. On s'accorde dans l'ensemble à considérer qu'il s'agit du Campā de la péninsule indochinoise mais ce n'est pas encore établi avec certitude et il faudra tenir compte des hypothèses contraires lorsqu'une étude plus approfondie de cette question sera menée ${ }^{3}$.

Le thème le plus souvent cité et dont la légende s'est emparé est celui de la Putri Cĕmpa, la "princesse du Cĕmpa ». On le retrouve principalement dans trois textes javanais et sundanais : le Sĕrat kanḍa Tanah Jawi ${ }^{5}$ et le Sĕjarah Bantën ${ }^{6}$.

Les variantes de ces divers textes en ce qui concerne cet épisode sont nombreuses, tant dans les détails du déroulement du récit que dans les noms des personnages. Mais le thème central reste le même? Le roi du Cĕmpa reçoit en son pays un voyageur, zélé musulman, venu du Moyen-Orient (Raja Pandita Mustakim/Makdum Brahim Asmara/ Pandita Arifin ${ }^{8}$ ). Ce dernier convertit à l'Islam le roi et tout son peuple. Le souverain hindouiste de Majapahit prend pour femme la fille musul-

(1) Drewes, 1968, p. 449. En 977, l'envoyé de Po-ni (Brunci) à la cour de Chine avait Pu pour patronyme (Hirth et Rockhill, 1911, pp. 157, 159, n. 13). Les Chinois eux-mêmes furent souvent ainsi envoyés à la cour des Ming (Chiu Ling-yeong, 1967, pp. 219-221).

(2) C'est à ces mêmes conclusions qu'est arrivé Kodo Tasaka, 1952.

(3) Rouffaer a tenté de situer le Cémpa des sources javanaises à Jeumpa, sur la côte nord d'Acch, mais il y voyait tout de même une déformation de Campä (Encycl. van Nederlandsch

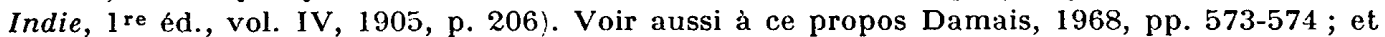
infra, p. 262, n. 1 et p. 263, n. 5,6, p. 267, n. 3. On se reportera encore à De Graaf et Pigeaud, 1974, pp. 10, 20-24, 112, 138, 170, 244-246, 271, 290-291, 318. kaṇla

(4) Brandes, 1920, pp. 222-224, où est donné un résumé des chants 396 à 399 du Sĕrat

(5) Olthof, 1941, pp. 18-31. C'est une traduction de la version résuméc en prose de J. J. Meinsma.

(6) Résumé dans Djajadiningrat, 1913, pp. 22-25. Commentaires et variantes des traditions sundanaises plus récentes, ibid., pp. 252-255, 263-264, 275-276.

(7) Outre les publications de textes précitées, une importante littérature a déjà été consacrée à la Putri Cĕmpa : Kern, 1938, p. 333 ; Cowan, 1939, pp. 121-126 ; Damais, 1968, pp. 573-574 ; Krom, 1931, pp. 452, 462-467 ; Maspero, 1928, pp. 228-229.

(8) On trouvera les variantes des noms telles qu'elles apparaissent dans les trois textes précités, dans l'ordre. Dans une tradition sundanaise plus récente, on trouve une généalogie de ce saint homme (qui y porte le nom de Samsu Tamres) : il est issu d'un inceste, sa mère ayant fauté avec son propre père, prince d'une ville arabe. Sa mère épouse par la suite un marchand du Cëmpa, venu en pèlerinage à la Mecque, nommé Juragan (capitaine) Kadiriyah ou Haji Dulkadir (Djajadiningrat, 1913, pp. 263-264). 
mane du roi (Darawati/«Putri Cĕmpa »/Andrawati). Le voyageur arabe a entretemps épousé l'autre fille du souverain, sœur de la "Putri Cěmpa ». Ils ont un enfant (Sayid Rahmat/Raden Rahmat/Raden Rahmat) qui, adulte, rejoint sa tante à $\mathrm{Java}^{1}$ et y reçoit du souverain de Majapahit le fief de Ampel (Ngampel) près de Surabaya. Il y convertit la population à l'Islam et sous le nom de Sunan Ampel sera par la suite cité parmi les neuf saints (wali sanga) à qui la tradition javanaise attribue l'introduction de l'Islam dans l'île. Son fils (Raden Bonang/Santri Bonang/ Pangeran Bonang) sera aussi l'un des wali sanga (sous le nom de Sunan Bonang) et sa petite-fille épousera son disciple Raden Patah, qui prendra le kraton de Majapahit et fondera le sultanat musulman de Demak $^{2}$.

La Putri Cĕmpa, dont on parle le plus, est en fait un personnage mineur de la tradition et ses enfants (lorsqu'elle en a, ce qui n'est pas le cas dans le Babad Tanah Jawi) ne jouent pas un bien grand rôle. C'est en fait de sa sœur, dont on ne connaît pas le nom, épouse au Cĕmpa du saint musulman, que sont issus deux des wali javanais.

Il n'est pas possible de dater avec précision ces événements, car les éléments fournis par les diverses traditions sont contradictoires. Seul le Sěrat kanda donne les chronogrammes des épisodes racontés : Raden Patah fonde Demak en 1326 śaka (1404 EG); la princesse Darawati (Putri Cĕmpa) meurt en 1320 śaka (1398 EG). Mais une tombe du cimetière de Trawulan, près du site de Majapahit, qui est considéré par la tradition orale comme la sépulture de la Putri Cĕmpa porte le millésime 1370 śaka (1448 EC) ${ }^{3}$. Par ailleurs, la tombe de Sunan Ampel, mort en 1467, se trouve encore dans la localité d'où il tire son nom. Les historiens s'accordent en général pour dater la prise du pouvoir par les principautés musulmanes du Pasisir, avec Demak à leur tête, de la fin du xve ou du début $d u \mathrm{XvI}^{\mathrm{e}}$ siècle. Mais la question ne semble pas encore résolue de savoir s'il s'agit là de la "chute " du kraton de Majapahit telle qu'elle est décrite par la tradition. Beaucoup d'éléments restent donc dans l'immédiat irréconciliables et, en ce qui nous concerne, on retiendra seulement le modèle proposé par ces traditions, qui fait intervenir dans le processus

(1) Rahmat est accompagné à Java par trois autres jeunes dont, selon le Babad Tanah Jawi, le fils du roi du Cëmpa, Raden Burereh, et son propre frère, Raden Santri. lls s'installent tous deux à Gresik. Une tradition sundanaise tardive, le Sejarah Banten rante-rante, affirme qu'après le départ de Raden Rahmat et de ses compagnons, le Cëmpa est dévasté par le roi de Koci. Djajadiningrat (1913, p. 254) n'a pas reconnu dans ce toponyme le Việt-nam (Giao-chi, cantonais /kawci/ > malais kuci ou koci; c'est ce nom qui est à l'origine du Cochin[china] des Portugais). C'est là un argument en faveur de l'assimilation du Cëmpa au Campā ; mais ce texte tardif est le seul à donner cet épisode et une autre version affirme pour sa part que le Cëmpa a été détruit par le roi païen de Sanggora (Singora/Songkhla ?).

(2) Le Hikayat Banjar propose aussi, dans un passage qui concerne Majapahit, un récit dont le déroulement est presque parallèle. La princesse n'y vient plus du Cĕmpa, mais de Pasai. C'est son frère Raja Bungsu qui se rend à Majapahit puis s'installe à Ampel où il convertit la population (Ras, 1968, p. 417).

(3) Rigg, 1851, p. 439 ; Brandes, 1886, p. 42. On rappellera qu'une tombe toute différente, puisqu'elle se trouve sur le site de Bukit Seguntang au sud de Sumatra, est aussi attribuée par la tradition orale à une Putri Cĕmpa. Elle n'a pas encore été déchiffrée (Damais, 1968, p. 584). 
d'islamisation de Java des personnages venus d'un pays que par hypothèse on considérera, jusqu'à plus ample informé, comme étant le Campā.

Un texte javanais de Cirebon, découvert plus récemment, fait aussi état à plusieurs reprises de relations entre La Mecque, le Cĕmpa et Java. Il affirme entre autres que l'enseignement de la loi coranique est donné au Cĕmpa par de célèbres maulana dont l'un a épousé la fille du souverain ${ }^{1}$.

Ce modèle semble bien confirmé par ailleurs dans des textes javanais moins connus, dans lesquels le Cĕmpa est toujours lié à la propagation de l'Islam² ${ }^{2}$ Les textes qui décrivent les généalogies des wali relèvent de la même tradition que les précédents, comme ceux qui donnent les généalogies des prophètes ou celles des rois javanais ${ }^{3}$. Mais il existe d'autres traditions qui lient le Cëmpa à la propagation de l'Islam dans l'Archipel. Un texte du Pasisir (Cirebon), le roman de Sela Rasa, raconte l'histoire de quatre princes du Cëmpa, nommés Selangkara, Sela Gada, Sela Cara et Sela Rasa, fils du roi Sek Beren. L'aîné Selangkara prend le pouvoir à la mort de son père et chasse ses trois frères ; ceux-ci vivent de nombreuses aventures, mènent des guerres contre les kafir (infidèles), qu'ils convertissent à l'Islam.

Un texte javano-balinais, le Kĕbo Mundar, mi-historique mi-légendaire, retrace l'introduction de l'Islam au Cĕmpa par un homme, fils d'un chien et d'une princesse issue d'une truie. Une deuxième version du même texte remplace le Cĕmpa par l'île de Lombok ${ }^{5}$.

Le roman encyclopédique javanais de Jatiswara établit encore des liens entre Cĕmpa et Islam ${ }^{6}$. Son récit commence au Cĕmpa : le roi Durnafi a un fils Ji Mortala et celui-ci deux fils ; l'aîné lui succède sous le nom de Aji Duta Samsu Mukdim. Le cadet, Ji Lalana, a deux fils :

(1) Texte javanais in : Atja (ed.), Tjarita Purwaka Tjaruban Nagari, Jakarta, 1972, pp. 76, 80. Une traduction indonésienne en a été publiée in : P. S. Sulendraningrat (ed.), Purwaka Tjaruban Nagari, Jakarta, 1972, pp. 13, 16-17.

(2) Les textes cités ici ont été relevés dans Pigeaud, 1967-1970 (cf. l'index, vol. III, p. 209, s.v. Cëmpa). Pigeaud semble tenir pour évidente l'équivalence Cëmpa = Campã ; mais il ne se prononce formellement en ce sens qu'une seule fois (vol. I, p. 226).

(3) Pigeaud, op. cit., vol. II, pp. 464, 489-492, 850, 859.

(4) Ibid., vol. I, p. 226 ; vol. II, p. 35 ; vol. III, p. 69 . Le poème commence ainsi, selon la traduction de Pigeaud : "What is offered in the poem is a tale from the history of Cĕmpa..." Un long résumé en est donné dans Vreede, 1892, pp. 198-200.

(5) Pigeaud, op. cit., vol. II, p. 204. Juynboll, 1911, p. 107. Je ne peux juger que sur les résumés des textes donnés par ces deux auteurs, mais l'attribution de ces deux versions d'un même texte - qui font intervenir un même personnage nommé Kĕbo Mundar - à deux pays différents, Lombok et Cĕmpa, ne donne-t-elle pas à penser que ce Cĕmpa pourrait être un toponyme de l'ile de Lombok ? A moins que la tradition ait établi un rapport entre deux régions qui ont été l'une comme l'autre islamisées très tardivement, dans le courant du $\mathrm{xVII}^{\mathrm{e}}$ siècle. Seul un javanisant pourra répondre à cette question par l'étude de ces textes.

(6) Pigeaud, op. cit., vol. II, p. 97. Vreede, 1892, pp. 327-330, en donne un résumé détaillé. Assez curieusement, vers la fin du récit, a lieu une guerre entre le Cëmpa et le Pratokal (Portugal ?) qui l'a envahi. Jatiswara attaque, entouré d'une armée de bêtes sauvages qui comprennent le langage humain. Le prince de Pratokal est vaincu et Jatiswara est intronisé sous le nom de Prabu Satmata. A quelques escarmouches près, il n'y a jamais eu de conflit entre le Portugal et le Campā, et peut-être faut-il encore voir là un argument en faveur d'une localisation du Cëmpa quelque part dans l'Archipel. 
Ki Sajati et Jatiswara qui, en tant que maulana, partent à Java. Jatiswara connaît de nombreuses aventures. Le récit est mêlé de leçons de théologie et de mysticisme islamiques ${ }^{1}$.

Accepter le rôle joué aux $\mathrm{XIV}^{\mathrm{e}}$ ou $\mathrm{xv}^{\mathrm{e}}$ siècles par ces personnages venus du Cĕmpa dans le processus d'islamisation de Java et de l'Archipel, ce n'est pas pour autant admettre que ce pays était islamisé. La conversion $d u$ roi et de son peuple, mise en avant par les textes cités, est contredite par ailleurs. Cette époque est encore relativement bien connue par l'épigraphie et l'archéologie, qui témoignent toutes deux des religions pratiquées à titre officiel par les souverains cam : abandon progressif du bouddhisme māhāyana dans le courant du xıII ${ }^{\mathrm{e}}$ siècle au profit du śivaïsme et $d u$ viṣnuisme ${ }^{2}$. Les premiers voyageurs européens confirment schématiquement ces données et semblent les élargir à l'ensemble de la population.

Marco Polo note la religion pratiquée dans les pays qu'il a visités, opposant bien, comme le feront beaucoup d'Européens après lui, les "idolâtres " aux musulmans (on sait que son témoignage est important pour les précisions qu'il donne sur les royaumes du Nord de Sumatra où l'islamisation en est à ses débuts). Il passe au Campā dans les années 1280 et, précise-t-il en ce qui concerne le roi et son peuple, "tous sont idolâtres ${ }^{3}$. Un demi-siècle plus tard, Frère Odoric de Pordenone visite à son tour le Campā, mais ne fait que confirmer l'existence du sacrifice des veuves, la satī : "Quand un homme marié meurt en ce pays son corps est brûlé et sa veuve avec lui $»^{4}$.

La religion d'État était donc à cette époque au Campā l'hindouisme. Mais il est permis de penser que des musulmans y évoluaient dans des cercles proches de la cour, qu'ils soient étrangers, comme le saint homme de la tradition javanaise, ou cam convertis, comme l'épouse musulmane de ce même personnage et sa sour la Putri Cĕmpa. D'après une histoire légendaire figurant dans un manuscrit cam du Việt-nam, un certain Pŏ klaŭ bārāhŭ - un dignitaire dont le nom n'est attesté nulle part ailleurs - aurait été élevé dans une famille musulmane et aurait par la suite refusé que son frère Pŏ sānimpat épouse la princesse brahmaniste

(1) On trouve encore ce toponyme attesté dans des traditions qui sont certainement prémusulmanes (bien que leur rédaction soit tardive) : le mythe du riz de Sri et de Sédana débute au Cĕmpa. Il apparait encore dans un traité juridique, le Sastra Paniti, qui précise qu'un fonctionnaire de la cour de Majapahit, nommé Udayaka, est descendant d'un brahmane de Cĕmpapura. On le trouve enfin dans des incantations, sous la forme Cëpah (Pigeaud, op. cit., vol. II, pp. 501, 518, 733).

(2) Boisselier, 1963, pp. 334-340, 359-369, où l'on trouvera résumées ces différentes données.

(3) "They have a king for themselves and their own people and their special language and are all idolaters." ("adorant ydola " dans le texte latin); Moulle et Pelliot, 1938, vol. I, p. 366, vol. II, p. LXII.

(4) Yule et Cordier, 1913, vol. 11, p. 166. Si l'on accepte de voir le Campā dans le Tawālisí d'Ibn Bațūta, comme Yamamoto a tenté de le démontrer d'une façon habile mais imparfaitement convaincante, on aurait un troisième témoignage de "l'idolâtrie " du pays, dans la première moitié du xıve siècle. Mais, outre l'identification même de Tawãlisī, on pourra contester comme Ferrand le seul fait qu'Ibn Bațūta se soil jamais rendu plus à l'Est que l'Inde (Yamamoto, 1936 ; Ferrand, 1914, vol. II, pp. 426-458). 
Pŏ saḥ ină $\bar{a}^{1}$. Ces milieux convertis, proches de la cour, comptaient très vraisemblablement des marchands parmi eux, qui fréquentaient les royaumes musulmans de l'Archipel ou même de l'Inde. Au Campā certainement, comme ailleurs dans le monde nousantarien, les négociants étaient en contact étroit avec le roi et sa cour, qui pouvaient contrôler les exportations et financer des voyages commerciaux ${ }^{2}$. A Majapahit même, les cimetières de Tralaya et de Trawulan, proches du site du kraton, avec leurs tombes musulmanes dont les premiers millésimes datent de la période la plus glorieuse du royaume $(1368,1376$, etc.) attestent sans aucun doute qu'il y a eu dès lors des conversions individuelles à l'Islam dans des milieux proches de la cour ${ }^{3}$.

La question qui se pose ici est de savoir s'il faut introduire une solution de continuité entre les témoignages moyen-orientaux et chinois, relatifs à la période qui va du viıI ${ }^{\mathbf{e}}$ au XI ${ }^{\mathbf{e}}$ siècles, et ceux fournis par les sources javanaises, qui ne peuvent être antérieurs au xrve siècle. Les premiers témoignages se réduisent aisément aux activités de colonies marchandes musulmanes étrangères dans les ports de l'Inde, du monde nousantarien (de Śrīvijaya au Campā) et de la Chine méridionale. Mais faut-il voir dans le second groupe de témoignages le prolongement des activités au Campā de telles colonies, ou doit-on considérer qu'ils s'intègrent dans le mouvement d'islamisation dont ils sont contemporains et qui ira en s'amplifiant à partir du xıII ${ }^{\mathrm{e}}$ siècle dans le monde nousantarien, jusqu'à sa conversion presque totale à la nouvelle doctrine au XviI e siècle? Le passage de l'une à l'autre de ces périodes constitue en fait le nœud du problème de l'islamisation de cette région du monde et il est loin d'être encore résolu'. L'état des connaissances sur le Campā ne permet pas d'amener des solutions nouvelles à ce problème.

En ce qui concerne plus particulièrement le Campā, les témoignages qui seront cités dans la deuxième partie de cet article, catégoriques dans leurs affirmations que l'Islam n'est pas implanté dans le pays avant la fin du Xvi $\mathrm{X}^{\mathrm{e}}$ siècle, peuvent $a$ priori paraitre inconciliables avec ce qui précède. Mais la plupart des auteurs cités, fréquentant en Asie du SudEst dès le xive siècle des états islamisés, ont pour habitude de leur

(1) Pŏ Jharma, 1978, p. 165, n. 195. Selon les chroniques, Pŏ sạ̣ inā est donnée comme la fille, ou la sceur du roi Pó kasil (ce roi aurait régné, selon les chroniques, entre 1421 et 1448, ou entre 1433 et 1460 ; Pŏ Dharma, 1978, p. 110 , n. 56a).

(2) Au Campā même, vers 1100 , la liaison du souverain avec les milieux commerçants est bien attestée par le mariage de sa fille à un marchand chinois qui avait séjourné dix années dans le pays (Mori, 1972, p. 12). Sur Ia participation des souverains nousantariens aux activités commerciales, cf. aussi Schrieke, 1957, vol. II, pp. 237-239 et notes pp. 386-387.

(3) Damais, 1957 ; 1968, pp. 570-577. On rappellera qu'une stèle arabe a de même été découverte au Cambodge à Phnom Bakheng. Elle ne contient qu'une formule pieuse et un verset du Coran et n'est pas datée. Son écriture serait "relativement moderne". Voir à ce propos la brève notice qui lui est consacrée dans le $B E F E O$, XXII, 1922, p. 160.

(4) Pour un récent historique de la question se reporter à Drewes (1968). L'auteur de cet article conclut en renvoyant les diverses théories dos-à-dos : "The investigation has been reopened, but without new data it seems that the results will as yet be scanty. Plus récemment, Al-Attas (1969, pp. 29-32) a défendu une thèse selon laquelle il n'y aurait aucune solution de continuite. 
opposer d'autres pays où l'Islam n'est pas religion d'État et n'est pas implanté en dehors des milieux commerçants cosmopolites. Il va de soi pour ces témoins que des musulmans peuvent être présents à divers titres dans ces états "idolâtres " ou "gentils" (qui font partie intégrante des réseaux commerciaux de la mer de Chine), sans qu'ils y jouent pour autant un rôle prépondérant, que ce soit par la qualité ou par la quantité des conversions à la nouvelle doctrine.

Ceci m'amène, avant de poursuivre, à tenter de définir, à la suite de ces témoins, ce que l'on entend par islamisation. Des définitions diverses en ont été données. Damais estime pour sa part qu'on ne peut en parler que lorsque le roi et sa cour ont officiellement adopté l'Islam et entrainent à leur suite le reste de la population ${ }^{1}$. Cette définition restrictive s'applique bien au processus qui s'est déroulé lors de la conversion des petits royaumes côtiers du Pasisir javanais. Mais le schéma inverse peut aussi être vrai, dans lequel la population marchande d'une ville portuaire est convertie à l'Islam et entraîne à sa suite le souverain qui dépend d'elle dans une large mesure. C'est ce qui s'est passé à Patani vers la fin du $\mathrm{XIV}^{\mathrm{e}}$ siècle $^{2}$, à Brunei au début du XVI ${ }^{\mathrm{e}}{ }^{3}$, à Makasar au début du XvII ${ }^{\mathrm{e}}$, et c'est ce qui se passera au Campā, pendant une courte période au moins, dans le courant du xvir siècle. On retiendra donc ici une définition plus large de l'islamisation, qui ne prendra en compte que le résultat de ce processus, sans tenir compte de l'ordre dans lequel il s'est déroulé : un royaume sera dit islamisé lorsque le souverain, sa cour et une notable fraction de la population seront convertis. Ceci dit, l'importance de cette date précise est toute relative : l'islamisation ne vient le plus souvent qu'entériner un état de fait et ne constitue qu'une étape dans un processus qui s'inscrit dans la longue durée.

$\mathrm{Au} \mathrm{xv}^{\mathrm{e}}$ siècle, c'est dans les sources chinoises que l'on trouve la première indication précise de la religion pratiquée au Campā, dans les récits recueillis lors des grandes expéditions des Ming, effectuées sous les ordres de Zheng He. Ma Huan, témoin oculaire puisqu'il a participé de 1413 à 1433 à trois de ces voyages, fournit dans son Y ingya shenglan (1433) des informations dignes de foi. Musulman lui-même, il prend soin de noter, pour d'autres États comme Aru, Lambri et Malaka, que les rois comme leurs peuples sont musulmans (huihui 回回). En ce qui concerne les ports du Pasisir javanais, il précise qu'une fraction seulement de la population est convertie. Au Campā, par contre, il note que le roi "suit la doctrine de shi 釋教 》, c'est-à-dire Sākyamuni, autrement

(1) Damais, 1968, p. 570 .

(2.) Teeuw et Wyatt, 1970, vol. I, pp. 3-5; vol. II, p. 221.

(3) Situation décrite d'une phrase laconique par Ruy de Brito, Capitaine de Malaka, en janvier 1514 : "Ho rey he cafere, os mercadores sam mouros " (Le roi est païen, kafir, les marchands sont musulmans). Lettre publiee dans Cartas de Affonso de Albuquerque, Lisboa, 1884-1935, vol. III, p. 92.

(4) Noorduyn, 1956. 
dit qu'il est bouddhiste'. Ma Huan confond bouddhisme et hindouisme (le māhāyanisme a disparu deux siècles auparavant), mais cette confusion est compréhensible de la part d'un Chinois musulman qui a pu se référer en outre à des sources antérieures, alors qu'il n'aurait pu manquer de reconnaître l'Islam s'il y avait été pratiqué à titre officiel. Gong Zhen, qui participa lui aussi à l'une des expéditions de Zheng $\mathrm{He}$, confirme les indications de Ma Huan dans son Xiyang fanguo $z h i(1434)^{2}$.

Le Sejarah Melayu, dans le passage qu'il consacre au Campāa, fournit indirectement des informations sur la religion qui y était pratiquée par l'un des fils du souverain (que ce texte nomme Indera Berma Syah [Indravarman?]), sa femme (Kini Mernam) et sa suite, dans la deuxième moitié du $\mathrm{xv}^{\mathrm{e}}$ siècle : lorsqu'après la chute de Vijaya, en 1471, ceux-ci se réfugièrent à Malaka, où régnait Sultan Mansur Syah (mort en 1477), ils durent se convertir à l'Islam4. C'est donc qu'ils n'étaient pas musulmans avant leur arrivée et on peut sans trop de risques en déduire que le souverain cam ne l'était pas non plus. De l'autre prince, son frère (Syah Palembang, ou Syah Pau Ling [Pŏ liang?], suivant les manuscrits) qui se réfugia à Aceh, il n'est rien dit concernant sa religion ${ }^{5}$.

La Suma Oriental de Tomé Pires est le premier document portugais à nous parler de la religion des Cam : "Le roi est gentil ${ }^{6}(\ldots)$. Il n'y a pas

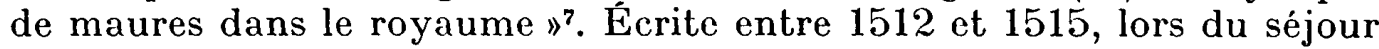
de son auteur à Malaka, la Suma Oriental constitue par son luxe de détails et son exactitude, un document exceptionnel. En ce qui concerne plus particulièrement les informations amenées par Tomé Pires sur la

(1) Feng Chengjun, 1935, p. 2. Mills, 1970, p. 79.

(2) Xiang Da, 1961, p. 2. Un texte plus tardif, le Xiyang chaogong dianlu de 1520, donne les mêmes indications. Mais il s'inspire pour une grande part des ouvrages précités et c'est donc vraisemblablement des informations vieilles d'un siècle qu'il fournit (Mayers, 1874-1875, p. 322).

(3) Cf. à ce propos l'excellent article de Marrison, 1951. Le contenu de ce passage du Sejarah Melayu permet sans conteste d'assimiler le Cempa dont il est fait mention au Campā. Le fait qu'en malais ce dernier soit désigné par Cempa $(=C \ddot{m} p a)$ constitue un argument de plus en faveur de l'équation : javanais Cĕmpa = Campā. On trouvera le texte du Sejarah Melayu (d'après le manuscrit Raffles, no 18) dans Winstedt, 1938 (pp. 134-137, pour les passages qui nous concernent) et le deuxième texle dans Shellabear, 1910 (cf. chap. 21, pp. 131-134). On se reportera aussi aux commentaires sur ce texte de Hooykaas, 1947, p. 235 ; à la traduction du manuscrit édité par Winstedt, par Brown, 1952, pp. 108-111 (rédité, sous forme de volume, à Kuala Lumpur en 1970). A. Marre, 1895, avait été le premier à signaler ces passages du Sejarah Melayu (Gerini, 1909, pp. 693-697, en avait fait état après lui).

(4) Sakaliannya disuroh masok Islam.

(5) La version Shellabear ajoute néanmoins que Shah Pau Ling fut à l'origine des souverains d'Aceh (ialah asal raja Aceh). A ma connaissance, aucun autre texte ne confirme cette affrmation. Et il est certainement très aventureux de se baser sur le seul Sejarah Melayu pour faire régner entre 1471 et 1505 une dynastie cam à Aceh, en expliquant en outre par là l'existence d'un fonds lexical commun aux deux langues : c'est pourtant ce qu'ont fait Gerini, 1909, pp. 696-698, et plus récemment Fatimi, 1963, p. 58. Sur les rapports entre les langues cam et acihaise, cf. les articles de Cowan, 1933, 1938, 1948, et Shorto, 1975.

(6) Gentil, c'est-à-dire paien, par opposition à musulman. Cette désignation groupait en général dans une même catégorie toutes les religions, sans distinction, qui n'étaient pas judéo-chrétienne et musulmane.

(7) Voir, pour le texte portugais, sa traduction anglaise et une longue étude sur son auteur : Cortesão, 1944 (vol. I, pp. 112-115 et vol. II, pp. 390-392). Pour une traduction française annotée du chapitre sur le Campā, cf. Manguin, 1972, pp. 39-42. 
religion des Cam, on doit rappeler, pour leur donner tout leur poids, combien les Portugais attachaient d'importance à la religion des peuples qu'ils fréquentaient, en distinguant bien les "gentils" de ceux qui suivaient, selon les propres termes de la Suma Oriental, "la fausse et diabolique doctrine de l'abominable, ignominieux et faux Mahomet, chef de toute la vaine religion mauresque "1. Tomé Pires précise, pour toute l'Asie du Sud-Est, la religion du roi comme celle du peuple, signale lorsqu'il le peut le pourcentage de ce dernier qui est converti à l'Islam (aux Moluques), la date d'introduction de la nouvelle religion (à Bornéo et aux Moluques), la présence de colonies musulmanes dans un pavs "gentil" (au Siam). Son affirmation catégorique en ce qui concerne le Campā, a pu lui être fournic à Malaka même par des négociants cam, dont il nous dit par ailleurs qu'ils se rendaient fréquemment en cette ville ${ }^{2}$.

Duarte Barbosa, qui rédigea à Goa sa description de l'Asie vers 1518, quelques années seulement après Tomé Pires, constitue une source moins sûre que son prédécesseur en ce qui concerne l'Asie du Sud-Est, où il ne se rendit jamais ${ }^{3}$. Il vient néanmoins confirmer Pires et mérite d'être cité : «(...) Une grande île ${ }^{4}$ de gentils que l'on nomme Champa et quj a un roi gentil (...)".

C'est vraisemblablement vers cette époque (dans la deuxième moitié $d u x{ }^{e}$ siècle) que l'art cam - statuaire et architecture fournit les derniers témoignages authentiques sur le śivaïsme pratiqué comme religion d'État. En dépit de la tradition cam qui fait de Pŏ ramō un roi de la première moitié du xvir ${ }^{\mathrm{e}}$ siècle $^{5}$, il faut voir dans le temple du même nom une construction datant du milieu du Xvi ${ }^{e}$ siècle $^{6}$. Le mukhalinga de Pŏ klaŭ garai, que Boisselier considère comme la "dernière idole authentique ", appartient à ce même "style de Pŏ ramō » et

(1) Cortesão, 1944, vol. I, p. 2, vol. II, p. 324.

(2) Bien que le lexte du Sejarah Melayu laisse entendre que les exilés cam s'étaient installés pour longtemps à Malaka, rien ne permet d'affirmer catégoriquement qu'il y avait encore en 1511 une colonie cam en cette ville. Tomé Pires, contrairement à ce que dit Marrison (1951, p. 97), ne parle pas d'un syahbandar chargé de veiller sur une telle communauté : il affirme seulement que cet officier est chargé, entre autres, des navires cam venus y commercer et dont lc chargement comme l'équipage tombent de ce fait sous sa juridiction (Cortesão, 1944, vol. I, p. 265, vol. II, p. 493).

(3) On trouvera le texte portugais de Duarte Barbosa, dont il existe de très nombreux manuscrits, dans : Colleção de noticias para a historia e geografia das nações ullramarinas (2e éd), t. II, Lisboa, 1867, pp. 237-388 (p. 373 pour celte citation); on le trouvera aussi dans libeiro, 1961 (p. 301). Une traduction anglaise en a été publiée par Dames, 1918 (vol. II, p. 208).

(4) Le: Campā est qualifié ici d'île ; Barbosa, qui recueille ses informations en Inde, a vraisenblablement mal interprété un terme dérivé de dvīpa qui désigne aussi bien une île qu'un territoire (cf. aussi le chinois zhou 洲).

(5) D'après les calcuis effectués par Pó Dharma (1978, p. 53), les neuf chroniques du Pānduranga donnent toutes pour date de règne de Pó ramō les années 1627-1651. Ceci confirme les supputations d'Aymonier.

(6) Boisselier, 1963, pp. 383-387.

(7) Ce mulhalinga est à séparer du sanctuaire du même nom (où il est abrité), qui, pour sa part, est daté de la fin du xire siècle (Boisselier, 1963, pp. 346-347). 
son installation pourrait même dater de la fin $d u x{ }^{e}$ ou du début du XVII ${ }^{\mathrm{e}}$ siècle $^{1}$.

Dans les dernières années du xvi ${ }^{\mathbf{e}}$ siècle, des projets de conquête de l'Asie du Sud-Est virent le jour, fomentés surtout par certains milieux de Manille liés aux Dominicains et, dans une moindre mesure, à Malaka sous l'impulsion de son évêque Dom João Ribeiro Gaio ${ }^{2}$. Ils nous ont valu, en raison surtout du rôle joué au Cambodge par une poignée d'aventuriers portugais et espagnols, une abondante documentation sur ce pays et ses voisins, largement utilisée par des auteurs comme Cabaton, Briggs, puis Groslier et Boxer ${ }^{3}$. Parmi les pays dont la conquête était projetée (Aceh, Java, Siam, Việt-Nam et même la Chine) figurait en bonne place le Campā : sa position clé sur la route maritime transasiatique faisait bien des envieux. Mais les raisons invoquées pour justifier de sa conquête n'étaient pas toujours aussi directes : son roi était traité de pirate, on lui reprochait de procéder à des sacrifices humains et à la récolte du fiel de ses sujets, et surtout de favoriser l'expansion de l'Islam dans son pays, alors que lui-même était païen.

L'ensemble des documents utilisés par les auteurs précités (Boxer excepté) avait été imprimé au Xvi ${ }^{\mathrm{e}}$ siècle et rédigé de seconde main sur la base de documents manuscrits dont une bonne partie est encore conservée à l'Archivo General de Indias de Séville. Parmi ceux-ci, il existe une description du Campā, rédigée en commun à Manille, le 7 décembre 1595, par un certain nombre de participants aux événements du Cambodge dont Diogo Veloso, qui signale avoir pris ses informations auprès de la colonie cam du Cambodge, Gregorio de Vargas Machuca, Blas Ruiz de Hernán Gonzalez et Francisco de Sagredo, qui précisent qu'ils ont pris leurs renseignements au Campā même, où ils avaient été faits prisonniers. En voici un extrait :

" [Il y a] en ce royaume de nombreux Mahométans et le roi désire et se fait un plaisir que l'on dise et enseigne la secte de Mahomet et, à cet effet, on y trouve de nombreuses mosquées. Il y aussi de nombreux autres temples de gentils. " ${ }^{4}$

Tous ces documents irnprimés et manuscrits précisent que le roi est "gentil " (c'est-à-dire non-musulman) et décrivent, qui les chars sur lesquels sont promenées les "pagodes" (les idoles), qui la satī des veuves

(1) Boisselier, 1963, p. 385 et fig. 238.

(2) Pour une etude d'ensemble de ces projets et de leurs motivations, on se reportera à Boxer, 1969.

(3) Cabaton, 1908a, 1908b, 1914a, 1914-1916 ; Briggs, 1949 ; Groslier et Boxer, 1958, donnent le récit le mieux documenté et le plus clair de ces événements. Boxer a récemment publié une description manuscrite du Campā de la fin du xvie siècle (1970).

(4) Archivo General de Indias, Séville : Patronato, legajo 25, ramo $3^{\circ}$, documento 58, fol. $7 \mathrm{v}^{0}-11 \mathrm{r}^{0}$. Il existe une copie du xvirre siecle de ce document au Museo Naval de Madrid (Colleccion Navarrete, vol. 18, fol. $302 \mathrm{r}_{-}^{\circ} 304 \mathrm{r}^{\circ}$ ). Le texte complet de ce document, avec un commentaire détaillé, sera publié dans une prochaine Élude cam. Sur le séjour de Ruiz et Vargas Machuca au Campā, cf. Cabaton, 1914a, pp. 104, 124 ; Blair et Robertson, 1903-1909, vol. IX, pp. 162, 173, vol. X, p. 237. 
des dignitaires du royaume ${ }^{x}$. Un autre document de l'époque permet de préciser ces informations et, en particulier, le rôle joué par Brunei dans la propagation de l'Islam :

"C'est une honte de considérer que parmi cette population, par la voie de Brunei et d'autres [pays] Mahométans, sont propagés le venin et le poison de leur fausse doctrine. ${ }^{2}$

Cet ensemble de documents ibériques de la fin du xvie siècle pourrait être qualifié de partialité, vu le but avoué qu'il se propose. Mais un premier document hollandais, légèrement postérieur, vient le confirmer avec d'intéressantes précisions. En octobre 1607, la flotte de l'Amiral Cornelis Matelief, de retour de Chine, mouilla une dizaine de jours au Gampā pour se réapprovisionner. Le roi résidait, semble-t-il, à Phan-ri ("il tenait sa cour au Nord du cap situé par $11^{\circ}$ de latitude"). Il était païen, mais "l'orangkaya " qui vint à bord était musulman ${ }^{3}$. Le "jeune roi ", frère de celui qui régnait, "aurait voulu embrasser la religion des maures, mais il n'osait le faire, à cause de son frère. "Le roi entretenait de bonnes relations avec la cour musulmane de Johor ${ }^{4}$.

Il semble donc bien que l'on soit ici à un stade crucial de l'introduction de l'Islam dans le pays. Selon une démarche habituelle, les dignitaires chargés des relations avec l'étranger sont convertis à la nouvelle religion (et certains marchands doivent l'être aussi). Le roi lui-même reste attaché au culte pratiqué par ses ancêtres, mais la génération montante (son jeune frère en l'occasion) est prête à se convertir. Les chroniques royales cam attestent bien que la succession par le frère puîné est habituelle au Campā. Si l'on suit ces mêmes chroniques, c'est semble-t-il Pŏ nit qui régnait alors, et c'est justement son frère Pŏ jaiparan qui lui succéda ${ }^{5}$. Mais il n'y est rien dit de la religion qu'il pratiquait.

La conversion d'une partie de la population cam à l'Islam est encore confirmée dans le courant du Xvil ${ }^{\mathrm{e}}$ siècle par diverses sources. Le Père Escalona OFM, de passage sur les côtes cam en 1640, voit son navire

(1) Cabaton, 1914a, pp. 23, 123 ; Blair et Robertson, 1903-1909, vol. X, p. 237. Le texte publié par Boxer (1970) est Je seul à ne décrire que des cérémonies hindouistes, sans faire mention des progrès de l'Islam. Les textes qui suivent confirment pourtant bien l'implantation de la nouvelle religion. Il faut peut-être conclure de ceci que Je texte donné par Boxer est antéricur à la compilation à Manille du recueil dont il fait partie, effectuée pour justifier les projets de conquête en Asie et datée de la fin du xvi ${ }^{\mathbf{e}}$ siècle. Le ton et le contenu diffèrent d'ailleurs très nettement de ceux de la description de Veloso et de ses compagnons.

(2) Lettre de Hernando de los Rios à Philippe II en date du 27 juin 1597 (Archivo General de Indias, Filipinas, legajo 18b).

(3) "... den Orangkaya ... de welke Mahometist was, doch de Coningh is Heydens...". Orang kaya désigne pour les Européens les dignitaires, les puissants des royaumes malais. L'expression elle-même est malaise, mais son équivalent est attesté en Cam (renseignement qui m'a été communiqué par Nara vija et Pó Dharma).

(4) Historische Verhael van de treffelijcke Reyse (...) door Cornelis Matelief de Ionge, inden jaren 1605, 1606, 1607, 1608, dans Begin ende Voortgang (...), s.l., 1646, pp. 119-121. Une traduction française en existe dans Recueil des Voiages (...) de la Compagnie des Indes Orientales (...), vol. III, Amsterdam, 1705, pp. 499-502.

(5) Aymonier, 1890, pp. 187-193. Quatre d'entre les chroniques étudiécs par Pŏ Dharma confirment que Pó nit régnait en 1607, mais cinq autres laissent à penser qu'il y aurait eu un interrègne cette année-là (Pö Dharma, 1978, pp. 40-41). 
attaqué par "de nombreux naturels de ce royaume, (...) gent mahométane $(\ldots) \|^{1}$.

Les renseignements pris en octobre 1665 par M. Hainques, premier prêtre des Missions Étrangères à parcourir la région, le confirment encore :

"La plupart des sujets naturels du Pays (...) estoient infectez des erreurs des Sarazins; mais néantmoins il ne remarqua dans les Villes qui se trouvèrent sur sa route aucune Mosquée, \& il remarqua que ceux principalement qui demeurent à la campagne sont si ignorans sur la secte dont ils font profession, qu'il y a beaucoup d'apparence que l'on pourroit aisément avec la grâce de Dieu les faire passer à notre sainte Religion, si on leur exposoit les Veritez \& la Morale. "²

Et encore en 1675 :

"Ils sont presque tous engagés dans le Mahométisme, dont ils n'observent que la moindre partie, parce qu'ils sont dans une grande ignorance de la plupart de ses maximes et de ses usages. $\|^{3}$

M. Mahot Mep est le premier prêtre à avoir été attaché à la mission du Campā, où il débarqua fin 1676 ; il y séjourna jusqu'à la fin 1678 , s'occupant essentiellement des chrétiens viêtnamiens. C'est de lui qu'émanent les premières informations précises sur l'extension de l'Islam et, surtout, il est le premier à mentionner la conversion du souverain cam :

"Le Roi et une partie du Royaume sont Mahométans, mais ignorants ; une partie est ensevelie dans le gentilisme. $»^{4}$

"Pour l'estat de la religion de ce Royaume, les Malayes qui sont Mahométans ont esté plus vigilants que nous ; ils s'y sont introduits et habitués ${ }^{5}$ en grand nombre, ils ont attiré le Roy et toute sa cour à suivre leur maudite secte. ${ }^{6}$

"Les Chiampois dont plus de la moitié sont Maures avec le Roy sans pourtant connaitre leur religion ; l'autre partie adore le ciel, et dans leurs maladies et accidents qui leur arrivent font des sacrifices aux diables pour en être délivrés. " ${ }^{7}$

«Le Roy qui est Mahométan (...) Le Roy et ses peuples Mahométans s'étant retirés vingt lieues au-dessus de la côte de la mer, dans les terres du dedans du Royaume (...) $\rangle^{8}$.

(1) Fr. Francisco de Jesus de Escalona, Relacion del viaje al Reino de la gran China..., publié in : A. Van den Wyngaert, Sinica Franciscana, Quaracchi, 1933, vol. II, pp. 303-304.

(2) Relation des Missions des Evesques Frangois aux Royaumes de Siam, de la Cochinchine, du Camboye, et du Tonkin, \& c, Paris, 1674, pp. 105-106.

(3) Relation des Missions et des Voyages des Evesques Vicaires Apostoliques, et de leurs Ecclésiastiques, ès années 1672, 1673, 1674 et 1675, Paris, 1680, p. 332.

(4) Archives de Ia Société des Mission Étrangères, Lettre de M. Mahot, juillet 1678, vol. 734, p. 293. Ce document et ceux qui suivent m'ont été aimablement communiqués par G. Moussay.

(5) Habitués \#, c'est-à-dire qu'ils y ont pris résidence.

(6) Asme, Relation de voyage de M. de Courtaulin (1678), vol. 734, p. 755.

(7) Asme, Lettre de M. Mahot, 1683, vol. 735, p. 199.

(8) Asme, Lettre de M. l'Évéque d'Héliopolis (F. Pallu), 29 novembre 1682, vol. 106, p. 104. Cf. aussi Launay, 1923, pp. 243-244. 
En 1685, M. Féret Mep témoigne à son tour de la religion musulmane du souverain et il reçoit des Missions Étrangères, à son usage personnel, un Coran'.

Les sources hollandaises viennent corroborer les documents émanant des missionnaires français, qui affirment la conversion du roi à l'Islam. Dans une lettre écrite par le souverain cam au Gouverneur Général à Batavia en 1680, il porte le titre manifestement malais de Paduka Seri Sultan ("Paducca Siry Sulthan "2). L'utilisation du terme sultan confirme bien son appartenance à l'Islam ${ }^{3}$. Cette lettre, que l'on ne connaît que par sa version hollandaise, traduite "mot à mot" du malais ${ }^{4}$, a été amenée en mai 1680 à la capitale des Indes Néerlandaises par deux ambassadeurs du roi cam, les "Orangkhaya Poeranja Mantri et Intche (ou Intie) Noman $»^{5}$.

Le souverain cam musulman qui règne pendant la période $1676-1685^{6}$ a trois frères résidant à Ayuthya, musulmans comme lui. L'un d'entre eux "est en grande considération " auprès de la cour ; il traduit "en langue de Chiampa " les lettres de Constance Faulkon et des pères des Missions Étrangères au roi, son frère? ${ }^{7}$. Mais ses deux frères prennent part à une révolte des musulmans d'Ayuthya en 1686. Le P. Tachard S.J. en a laissé le récit :

"Trois frères fils du feu Roy de Champa qui se sauvèrent ici à l'avènement de leur Frère aîné à la couronne, de crainte d'en recevoir quelque

(1) Asme, Relation des Missions de Chine, Tunkin, etc., année 1686, vol. 855, p.556; État des Missions de Chine, Tonkin, etc., en 1686, vol. 879, p. 565 ; Mémoire de ce qu'on a envoyé en Cochinchine... le 20 may 1686, vol. 736, p. 111. Cf. aussi Launay, 1923, p. 352.

(2) Dagh-Register gehouden int Casteel Batavia..., Anno 1680, Batavia, 1912, pp. 242, 252-253. Le contenu de cette lettre, d'ordre commercial, a peu d'intérêt pour le présent travail ; le document complet sera donné à une autre occasion.

(3) Ce titre est attesté sous la forme sūlātān dans la généalogie de la famille princière de Pall canar (Durand, 1906, pp. 288-289). On ne le trouve pas dans les chroniques royales traduites par Aymonier (1889 et 1890).

(4) Il est normal que le roi cam, vu ses contacts avec les Malais et la colonie malaise qui résidait en son pays, utilise la langue de relation de l'Asie du Sud-Est maritime. Le roi cambodgien lui-même, au $\operatorname{xvı}^{\mathrm{e}}$ siècle, s'exprimait en cette langue et l'utilisait, avec le portugais, dans sa correspondance avec les Hollandais (Cabaton, 1914 b, p. 47 du tiré à part).

(5) Les noms qui sont attribués par le texte hollandais à ces deux orang kaya cam ne sont que partiellement identifiables. "Poeranja mantri ": |puraña/ est difficile à reconstituer; il semble bien qu'on doive le couper en deux et y voir le titre nousantarien $p u / m p u$ (cam pŏ), suivi d'un anthroponyme ou d'un teknonyme. On peut le rapprocher du vieux javanais $p u$ rañjan ou bien encore de pu ranyā, tous deux attestés au ix ${ }^{e}$ siècle; dans les deux cas, $p u$ doit être suivi d'un anthroponyme (Damais, 1970, pp. 227, 239). On peut aussi penser au sanskrit rājan, précédé du titre nousantarien, ce qui donnerait pu/pó rājño mantrí. "Mantri " ne fait pas difficulté : il s'agit bien sûr du titre qui est à l'origine du "mandarin " des langues européennes : malais menteri/mantri, cam mantrī (skr. mantrin). L'ensemble de l'expression peut encore être comparée, dans sa forme, à Pŏ ganvăr matrĭ, " seigneur chef des ministres ", nom attribué au Siva du fronton du temple de Phan-rang (Dictionnaire Cam d'Aymonier et Cabaton, s.v. muntri). "Intche (ou Intie) Noman": le malais ence', titre très courant, devrait normalement être suivi d'un anthroponyme; mais Noman, en malais, ne semble pas attesté comme nom de personne. On ne peut ici que le rapprocher du balinais Nioman.

(6) D'après les calculs de Pó Dharma (1978, p. 49, 53, 135), c'est Pó saut qui règne entre 1660 et 1692 .

(7) Asme, Lettre de Mgr Laneau, 3 juillet 1686, vol. 860, p. 123. 
mauvais traitement. De ces trois frères, il y en a un auprès du Roy du Siam, qui est officier de sa maison, et qui n'étoit point de la faction, et les deux autres vivaient en personnes privées. (...) [Le plus jeune] s'aboucha avec un Capitaine Malais, aussi natif du Champa, homme de courage, de tête et de lettres. (...) et ce fut luy, avec un de leurs Prêtres, qui conduisit toute l'affaire."

Cette insurrection était préparée conjointement par les Makasar, les Malais et les Cam musulmans d'Ayuthya, et menée à la sollicitation des princes cam "qui avoient résolu de couronner le plus jeune des frères de Sa Majesté, et de lui proposer ensuite le turban [la conversion à l'Islam], ou la mort ", avant, dit le P. Tachard, de placer sur le trône l'un d'entre eux'.

Entre 1607, date à laquelle on sait que le roi cam est encore brahmaniste, et 1676 , où l'on apprend qu'il est musulman, près de soixante-dix années se sont écoulées, pendant lesquelles aucun témoignage ne nous précise la religion du souverain. Les chroniques royales cam, selon les versions, énumèrent sept ou neuf souverains pendant cette période ${ }^{2}$, et c'est donc sous le règne de l'un d'entre eux que le Campā a été, à proprement parler, islamisé.

Que nous apprennent les sources et les traditions orales cam pour cette période? La tradition veut que le fameux roi Pŏ ramō (1627-1651, selon Aymonier, confirmé par Pŏ Dharma), fin politique, soit parvenu à mettre fin aux querelles entre Cam bañ̄ et kafir et soit à l'origine de la présence des prêtres banī lors de certaines cérémonies brahmanistes et de prêtres brahmanistes à la mosquée pendant la célébration du ramadan $^{3}$. Mais la tradition dit aussi qu'il y a eu crémation à sa mort et elle conserve le souvenir de la sat̄̄ des veuves du souverain ${ }^{4}$. Il semble néanmoins que l'une des épouses de Pŏ ramō ait été musulmane ${ }^{5}$. Par ailleurs, le manuscrit măbalai étudié par Aymonier cite bien, pendant le XVII ${ }^{\mathrm{e}}$ siècle, un roi auquel les Cam banī (Cam musulmans) "doivent leur bonheur ». Pŏ thaut "convoquait sans cesse les tourterelles (le peuple) pour leur faire embrasser l'Islam ", dit encore ce manuscrit;

(1) Second voyage du Père Tachard... au Royaume de Siam, Paris, 1689, pp. 97-101. L'alliance entre les colonies cam et makasar est confirmée dans une lettre du P. Morelli OFM, de 1686, publiée in : M. da Givezza et T. Domenichelli, La Palestina e le rimanenti missioni francescane, vol. I, Firenze, 1890, p. 751. La colonie cam d'Ayuthya est signalée pour la première fois en 1662 sans que sa religion soit précisée (Relation du Voyage de Monseigneur l'Evesque de Beryte... par M. de Bourges, $3^{\mathrm{e}}$ ed., Paris, 1683, p. 112).

(2) Pŏ Dharma, 1978, p. 53 ; Aymonier, 1890, pp. 151-152. Ceci, bien sûr, si l'on accepte les supputations de ces auteurs basées sur ces chroniques. Mais si le détail des dates ainsi fixées avec précision peut être discuté, un simple calcul de générations permet de situer grosso modo certains souverains au Xvir ${ }^{\circ}$ siècle.

(3) Po Dharma, 1978, p. 127, n. 90b.

(4) Durand, 1903b, pp. 601-603. La sati des veuves est de même attestée par quatre chroniques royales (Pó Dharma, 1978, pp. 124-125). Les indications qui suivent, tirées des textes et de la tradition orale cam, ont été fournies, sauf indication contraire, par Aymonier (1890, pp. 170-182). Certaines des informations que ce dernier a recueillies et qui émanent des Cam du Cambodge doivent être acceptées sous toutes réserves (Pó Dharma, 1978, p. 44).

(5) Pó Dharma se réfère à ce propos à l'expression utilisée dans une chronique en ce qui concerne l'épouse byā sumūt de Pó ramo (Pó Dharma, 1978, pp. 126-127). 
mais il était kafir, affirme la tradition orale. La tradition cite aussi un certain roi Pŏ klaŭ gahul, dont la tombe est conservée à la grande dune (gahul) de Phan-ri, où l'on enterre aujourd'hui encore exclusivement des musulmans. Cette tradition, précise d'ailleurs Aymonier voudrait qu'aucun roi n'ait été musulman, sauf peut-être un certain Pŏ li (Alì?) qui n'est pas mentionné dans la chronique. Dans l'enclos funéraire de Pŏ nraup, successeur de Pŏ ramō, on trouve encore les tombes de deux de ses femmes, dont la tradition dit qu'elles étaient l'une kafir et l'autre banīi.

Il est donc encore exclu de pouvoir tirer des renseignements précis des chroniques royales et de la tradition cam. A leur lecture et à celle de leurs commentateurs appuyés sur la tradition orale, il se dégage tout de même une constante : pendant le Xvir ${ }^{\mathrm{e}}$ siècle, la progression de l'Islam a constitué un phénomène qui a marqué les esprits. Ceci cadre bien avec ce que nous ont appris les sources européennes.

Un témoignage hollandais de 1644 pourrait tout de même laisser entendre que le souverain n'était pas encore converti à cette date. En effet, en juillet de cette même année, lors de la réception de Domkes au " camp de chasse " du roi à Cana, ce dernier confia aux Hollandais son opinion sur l'accession au trône du roi du Cambodge Barom Reachea VI (Paramarājā) qui, converti à l'Islam, avait pris le titre de Sultan Ibrahim en 1642; accession, on le sait par ailleurs, qui a été favorisée par l'action des communautés cam et malaises du Cambodge ${ }^{2}$. Le roi cam leur dit qu'Ibrahim avait traitreusement agi, et qu'il ne comprenait absolument pas ce procédé. Lui-même était un grand allié du précédent roi et depuis l'épouvantable meurtre commis par Ibrahim en 1642, il considérait son alliance comme rompue ${ }^{3}$. Craignait-il de voir des événements du même ordre - un coup d'état des musulmans - se produire chez lui? Condamnait-il seulement la manière douteuse de l'accession au trône du nouveau roi khmer et les meurtres des Hollandais en 1643 ? Si l'on peut identifier ce roi cam au Pŏ ramō de la chronique (1627-1651 selon Aymonier et Pŏ Dharma) que la tradition dit brahmaniste, il semble bien qu'il y ait une nouvelle présomption, bien faible il est vrai, en faveur de son maintien dans cette religion.

C'est donc, en tout état de cause, entre 1607 et 1676 que le roi cam s'est converti à l'Islam et, si ces présomptions se confirment, après 1644 . De la religion pratiquée par les souverains qui ont succédé à ce ou ces rois, les sources postérieures dont je dispose ne disent malheureusement rien. On n'a plus beaucoup parlé du Campā aux $\mathrm{XVIII}^{\mathrm{e}}$ et $\mathrm{XIX}^{\mathrm{e}}$ siècles, avant que les premières études sur ce pays ne voient le jour, sous la plume de

(1) Parmentier, 1908, p. 49.

(2) Mak Phoeun, 1975, pp. 52, 212, 413-414. Les chroniques nomment ce roi " l'Auguste Rāma qui est entré dans la religion (musulmane) "(Rāmmā Gūla Sāsna), ou encore "Le Cam * (d'après Cabaton, $1914 b$, p. 53 du tiré à part).

(3) Cité d'après des documents d'archives par Van Dijk, 1862, p. 331.

(4) Des recherches dans les archives de la Compagnie des Indes Néerlandaises permettraient peut-être de préciser ce point. Pendant cette période, les relations ont été fréquentes entre la VOG et le Campā (Buch 1936-1937). 
Moura, puis d'Aymonier1. Tout au plus doit-on citer encore le récit de la visite de la frégate française La Galathée à Phan-ri en 1720 , où il est affirmé que le "Mahométisme » est l'une des religions dominantes et que "l'idolâtrie y règne aussi " ${ }^{2}$. Un siècle plus tard, ce sont à nouveau les prêtres des Missions Etrangères qui nous fournissent les premières descriptions assez détaillées des religions pratiquées au Campā, parmi lesquelles l'Islam figure en bonne place. Mais rien n'est dit de la religion du souverain ${ }^{3}$.

L'étude stylistique de la statuaire vient confirmer les sources écrites. Aux dernières statues cam se substituent alors les $k u t$, stèles funéraires qui témoignent selon Boisselier "d'une volonté délibérée de faire disparaitre la figure humaine ". L'influence de l'Islam n'est sans doute pas à exclure ; "les kut de la région de Phan-ri ne sont pas sans rapports avec des tombeaux musulmans à Java, dont nous retrouvons encore le souvenir dans la composition de certaines stèles des cimetières chams musulmans du Cambodge. $\nu^{4}$

On en est donc réduit à une unique certitude : l'un, au moins, des souverains cam s'est converti à l'Islam dans le dernier quart du Xviı ${ }^{\mathbf{e}}$ siècle. On peut donc considérer qu'à cette époque le Campā était islamisé, mais sans pour autant affirmer que cette islamisation ait été définitive, puisqu'on ne sait pas si les rois suivants ont continué à professer la doctrine nouvelle. Le processus en cours ayant par ailleurs été interrompu pour des raisons qui lui sont extérieures (annexion par le Việt-nam), on doit considérer en outre que l'islamisation n'a été que partielle. Le souverain et une part importante de la population sont convertis : plus de la moitié vers 1676 d'après M. Mahot MEP, un tiers seulement au $\mathrm{xx}^{\mathrm{e}}$ siècle dans le Bình-thuânn (l'émigration vers le Cambodge serait-elle à l'origine de cette diminution?). Comme dans le monde nousantarien, le reste de la population aurait vraisemblablement suivi : si l'on compte les Cam émigrés au Cambodge, c'est près de $80 \%$ de la population cam totale qui est convertie ${ }^{5}$.

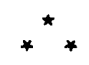

Il n'est pas indifférent, pour mieux appréhender le processus par lequel le Campā a reçu l'Islam, de s'étendre sur les rapports de ce pays avec les états islamisés voisins et avec les colonies musulmanes résidant

(1) Moura, 1883, vol. I, pp. 458 sq. Pour les œuvres d'Aymonier, cf. la bibliographie qui en est donnée par G. Cœdès dans sa notice nécrologique (BEFEO, XXIX, 1929, pp. 542-548).

(2) D'Après de Mannevillette, Le Neptune Oriental, Paris, 1775, p. 148 sq. J'ai cherché aux Archives Nationales (section Marine), avec l'espoir d'y trouver plus de précisions, le journal de bord de La Galathée, sur lequel de Mannevillette a dû prendre ses informations. Il ne s'y trouve malheureusement pas.

(3) Asme, lettre de M. Grillet, 18 juillet 1803, vol. 747, p. 79 sq. ; lettre de M. Tabard, 25 février 1829 , ibid., p. 1030 sq.

(4) Boisselier, 1963, pp. 391-393.

(5) Moura, 1883, vol. I, p. 494. Maspero, 1928, p. 13. Ces chiffres sont sujets à caution car, parmi les Cam du Cambodge, étaient souvent recensés des Malais ou des musulmans d'Insulinde. 
dans des pays non islamisés, en reprenant ce qui a été dit plus haut et en le précisant, si possible, par de nouveaux témoignages.

On sait déjà qu'une colonie cam s'était installée à Malaka à la fin $\mathrm{du} \mathrm{Xv}^{\mathrm{e}}$ siècle. Les Cam avaient de même au Cambodge une colonie importante dont les menées étaient liées à celles de la colonie malaise, beaucoup plus faible numériquement. La collusion de ces deux colonies a mis sur le trône khmer Sultan Ibrahim en 1642; mais elle était déjà évidente en 1599, lorsque les rebelles, ayant à leur tête le Cam Pŏ rat et un Malais dont on ne connaît que le titre laksamana, après avoir détruit une expédition espagnole, avaient assassiné le jeune roi khmer, pour se réfugier enfin au Campāi. On a vu au Siam, en 1686-1687, des Cam musulmans comploter encore, mêlés aux colonies malaises et makasar.

Par ailleurs, les bonnes relations entre le roi du Campā et le sultan de Johor étaient mentionnées par les Hollandais en 1607. On sait aussi qu'en 1594 , le roi cam avait envoyé des secours pour assister Johor dans son combat contre les Portugais de Malaka².

Les Cam ont eu leur part du commerce maritime Sud-Est asiatique et ont continué de participer à ses échanges, malgré l'affaiblissement progressif de leur pays. La tradition de Java Ouest fait intervenir un certain Juragan (capitaine) Kadirinyah, ou Haji Dulkadir, capitaine du Cĕmpa en pèlerinage à La Mecque ${ }^{3}$. Dans le Sejarah Melayu, au chapitre où il est question de l'institution du cérémonial de la cour nouvellement convertie de Malaka, les seuls étrangers à être nommément cités pour l'attribution des places dans la salle d'audience sont des capitaines cam de haut rang (nakhoda Cempa yang pilehan ${ }^{4}$ ). Près d'un siècle plus tard ${ }^{5}$, il y est fait mention d'un autre nakhoda cam, ayant pour nom Saidi Ahmad, qui aida Hang Nadim à enlever Tun Teja, la fille du bendahara de Pahang, et qui la ramena sur son navire pour que Sultan Mahmud de Malaka puisse l'épouser. Il reçut en récompense, outre de riches présents, le titre de Tun Setia 'diraja (version Winstedt) ou de Syah Andeka Menteri (version Shellabear) et l'autorisation de se tenir au pied $\mathrm{du}$ trône du Sultan avec les hérauts ${ }^{6}$. Le nom que porte ce capitaine de navire fait de lui un musulman. L'un des syahbandar de Malaka, à l'arrivée des Portugais, était chargé, entre autres marchands, de ceux venus du Campā et l'on retrouve des navires cam à Pahang et au Siam?. Une quarantaine d'années plus tard, Fernão Mendes Pinto - l'auteur de la fameuse Peregrinaçam - signale à Patani «de nombreux Maures

(1) Mak Phoeun, 1975, pp. 83-84, 99-100 ; Piat, 1974, p. 44 ; Antonio de Morga, Sucesos de las Islas Filipinas (éd. Retana), Madrid, 1910, pp. 146-148, 278; Moura, 1883, vol. II, p. 54, qui cite avec une date erronée la chronique royale khmer ; Briggs, 1950, p. 27.

(2) Blair et Robertson, 1903-1909, vol. X, p. 239.

(3) Supra, p. 261, n. 8.

(4) Shellabear, 1910, p. 64 ; Winstedt, 1938, p. 85 ; Brown, 1952, p. 55.

(5) Sous Sultan 'Abdu'l-Jamil de Pahang (ca. 1497-1511) et Sultan Mahmud de Malaka (1488-1530), donc entre 1497 et 1511, date de la prise de la ville.

(6) Shellabear, 1910, pp. 184-190; Winstedt, 1938, pp. 170-173 ; Brown, 1952, pp. 145-148.

(7) Supra, p. 268, n. 2; Cortesão, 1944, I, pp. 103, 113, 268. 
cam et minangkabau $\|^{\mathbf{1}}$. Un peu plus tard, il combat une flotte pirate, commandée par le gujrāti Khoja Hasan, dont l'équipage est composé de "maures luçons, bornéans, javanais et cam "². A la même époque, les Cam fréquentent les ports de Makasar, où ils rencontrent des gens de Pahang, de Patani, de Johor et du pays Minangkabau ${ }^{3}$.

Les informations tirées des traditions malaises et sundanaises ou fournies par Fernão Mendes Pinto ne doivent peut-être pas être toutes prises à la lettre ${ }^{4}$, mais, en tout état de cause, elles mettent en évidence avec les témoignages qui suivent la présence de marchands cam musulmans parmi ceux, de toutes nationalités, qui sillonnaient les mers de l'Asie du Sud-Est et formaient dans ses ports d'importantes colonies étrangères. Ces marchands d'origines diverses, en retour, fréquentaient les ports cam, d'où étaient surtout exportés le fameux bois d'aigle (kelambak), l'ivoire et l'ébène. En 1637 encore, parmi les marchands étrangers de Manille (Malais, Javanais, Chinois, Japonais et d'autres venus des Moluques, de Bornéo et du Moyen-Orient) on trouvait aussi des Cam5. En 1668, le sultan de Banten envoya au Campā l'un de ses navires pour y commercer ${ }^{6}$. En 1680 , on a vu que deux ambassadeurs du sultan du Campā arrivèrent à Batavia; ce souverain envoya encore en 1682 deux de ses navires commercer à Malaka?. Les Hollandais avaient des relations suivies avec le Campā et les Portugais aussi, dans une moindre mesure ${ }^{8}$. Les Japonais, pour leur part, fréquentèrent assidûment le pays à la fin du Xvie et au début du XviI ${ }^{\mathrm{e}}$ siècle $^{9}$.

Ces témoignages sur les rapports du Campā avec le reste de l'Asie éclairent deux points essentiels de l'histoire des derniers siècles d'existence du royaume. Ils contredisent d'abord catégoriquement le mythe de la disparition du Campā de l'histoire de l'Asie du Sud-Est après la chute de sa capitale Vijaya en 1471. "C'est ici que finit l'histoire du royaume de Champa » concluait Maspero dans le dernier chapitre de la

(1) " $\mathrm{m}^{\mathrm{t} \rho \text { s }}$ mouros Champos e Menãcabos" (Ayres, 1904, p. 79). On sait la conflance mitigée qu'il faut accorder dans le détail aux épisodes picaresques de la Peregrinaçam, écrite de mémoire après le retour de son auteur au Portugal et publiée un demi-siècle plus tard, après sa mort, en 1614 (ouvrage qui n'a en outre jamais eu de prétentions historiques). Mais ces objections ne s'appliquent pas au document cité ici, copie d'une lettre originale de Fernão Mendes Pinto, en date du 20 novembre 1555, alors qu'il faisait partie de la Compagnie de Jésus. Le chapitre 220 de la Peregrinaçam, qui mentionne ce passage à Patani en 1555, omet la rencontre de son auteur avec ces "Maures".

(2) "Mouros Lusoẽs, \& Borneos, \& Iaos, \& Champaas " (Peregrinaçam, chap. 57, éd. Costa Pimpão, Porto, 1944-46, II, p. 113).

(3) Noorduyn, 1956, p. 248.

(4) D'autant moins que le même épisode est attribué dans le Hikayat Hang Tuah au héros lui-même (qui devint le premier laksamana de Malaka, et non pas à Hang Nadim (qui fut, lui aussi, laksamana). Quant à notre nakhoda Cempa, il n'y apparaît pas (éd. Shellabear, 1914 , vol. II, pp. 191 sq.).

(5) Mémorial de Gran y Monfalcon, publié dans Blair et Robertson, 1903-1909, vol. XXVII, p. 82.

(6) Coolhaas, 1968, vol. III, p. 638

(7) Dagh-Register ... Anno 1682, Batavia, 1931, p. 1463.

(8) Buch, 1936-1937 ; Manguin, 1972, pp. 167-168, 236, 244, 247.

(9) Péri, 1923, pp. 31, 34, 52. 
seule histoire d'ensemble qui ait été consacrée à ce pays ${ }^{1}$. Or ce royaume était encore assez fort à la fin $\mathrm{du}_{\mathrm{XvI}}{ }^{\mathrm{e}}$ siècle pour envoyer de l'aide à Johor et ses marchands ont continué tout au long $\mathrm{du}$ XVII ${ }^{\mathrm{e}}$ siècle à fréquenter les ports de l'Asie du Sud-Est. L'effacement de ce pays de la scène sud-est asiatique devait en fait être très progressif et le coup le plus sérieux lui fut vraisemblablement porté lorsqu'entre 1691 et 1697 les Viêtnamiens - entérinant un état de fait - - en firent une province du domaine des Nguyễn (le Bình-thuận) et que les derniers ports cam passèrent sous leur contrôle ${ }^{2}$. Isolés du reste de l'Asie, retirés à l'intérieur des terres, les Cam ont survécu dans un milieu qui leur est hostile, sous l'autorité d'un roitelet nommé par Huê et contrôlé sur place par l'administration viêtnamienne.

Ces témoignages éclairent par ailleurs le processus même de l'islamisation du pays. Dès après la fondation de Malaka, le rôle qu'y ont joué les Malais apparait comme prépondérant ${ }^{3}$ : celui de l'État même de Malaka (puis de Johor où il se réfugie après sa chute), mais aussi celui des turbulentes colonies malaises, au Cambodge surtout, qui ont été en contact permanent avec des colonies d'émigrés cam qui se convertirent à leur contact; de même l'immigration malaise au Campā semble avoir eu sa part d'influence ${ }^{4}$. Il semble bien que les Cam, comme les Malais, soient de rite sunnite shāfi'ite, et, comme eux, qu'ils aient gardé des nuances de dévotion shì'ite ${ }^{5}$. 'Alī, Hasan et Husain sont particulièrement révérés et invoqués ${ }^{6}$. Si l'on ajoute à cela les relations établies de longue date avec le reste d'une Asie du Sud-Est nousantarienne progressivement islomisée, on comprendra que les Cam aient pu tenter par ce biais de se raccrocher à ce monde dont ils faisaient déjà culturellement partie, dans la position de plus en plus isoléc qui était la leur, au fur et à mesure de leur absorption par le Việt-nam et de la déliquescence de

(1) Maspero, 1928, p. 240. Boisselier (1963), dans un ouvrage dont le champ de recherches est plus vaste que la statuaire, est le seul à s'être intéressé aux derniers siècles de l'histoire cam. Les recherches d'Aymonier et surtout de Po Dharma portent sur des chroniques menant jusqu'en 1822 .

(2) Đại-nam thực-lục tiển-biên, VII, 4a-13b (trad. de Hà-nội, vol. I, 1962, pp. 147-153). Đai-nam chinh-biên liệt-truyện, I, 33, 21b-22a. Sur le récit de ces évênements par les Cam, voir Pó Dharma, 1978, pp. 53, 60, 64, 131.

(3) C'est un processus similaire qui s'est déroulé à Cólèbes-Sud : installation de commerçants malais, nombreux déjà au début du xvi siècle, et islamisation des divers souverains dans la première décade du xvire siècle (Noorduyn, 1956).

(4) L'influence malaise est apparente dans la littérature cam : on y trouve par exemple des textes comme le Dēuāmanō et l'Inrāpatrā qui sont le pendant des Hikayat Dewa Mandu et Inderaputera malais (cf. les traductions commentées de ces deux textes cam, respectivement par G. Moussay et Nara Vija ; diplômes EPHE, IVe section. Les positions de thèse de ces deux auteurs ont paru dans l'Annuaire de la IVe section de l'EPHE, 1975-76, pp. 1085-1091). Le rôle joué dans la littérature cam par le roi Nushirwan semble bien aussi être un apport malais (Durand, 1907). On citera aussi la traduction malaise interlinéaire d'un texte religieux arabe, copiée par un imām malais originaire de Châu-đôc en 1893, "dans le dessein de ramener à un islamisme plus éclairé ses coreligionnaires chams de l'Annam * (Cabaton, 1904, p. 115).

(5) L'étude reste à faire de la part des influences des rites divers dans le monde nousantarien; elle pourrait aider à déterminer l'origine de l'Islam qui y est pratiqué. Sur les survivances shī'ites dans l'Archipel, cf. entre autres Snouck Hurgronje, 1906, I, pp. '202 sq. et, pour une présentation récente de cette question, Baroroh Baried, 1978.

(6) Cabaton, 1927, p. 540. 
leur État. On voit qu'en parlant "d'immigration malaise ", Cabaton avait bien vu le rôle joué par cette ethnie ; mais la notion d'immigration (en péninsule indochinoise), si elle est confirmée par les sources citées plus haut, est néanmoins trop restrictive, la participation active aux réseaux du monde maritime Sud-Est asiatique ayant certainement eu une part essentielle dans le processus d'islamisation du Campā.

Le fait que le Campā ait été islamisé pendant son dernier siècle d'indépendance explique encore la soi-disant "dégénérescence " de cette religion. Les témoignages des missionnaires français sont unanimes pour reconnaître dès le $\mathrm{XVII}{ }^{\mathbf{e}}$ siècle le côté sommaire de la religion pratiquée par les Cam. Or, au Xvir e siècle, ce qui restait du Campā était définitivement coupé du reste du monde musulman et se voyait même partiellement imposer une culture sinisée qui lui était totalement étrangère ${ }^{1}$. L'Islam, implanté de façon trop précaire au Campā, n'aura pas pu y connaitre l'approfondissement et l'intensification qui sera le sien dans une bonne partie du monde nousantarien.

\section{ABRÉVIATIONS}

ASME : Archives de la Société des Missions Étrangères de Paris.

BEFEO : Bulletin de l'École Française d'Extrême-Orient.

BKI : Bijdragen van het Koninklijk Instituut.

BSEI : Bulletin de la Société des Etudes Indochinoises.

$J A \quad:$ Journal Asiatique.

JMBRAS : Journal of the Malayan Branch, Royal Asiatic Society.

$N B G \quad$ : Notulen van het Bataviaasch Genootschap.

PEFEO : Publications de l'École Française d'Extrême-Orient.

RMM : Revue du Monde Musulman.

TBG : Tijdschrift van het Bataviaasch Genootschap.

$V B G \quad$ : Verhandelingen van het Bataviaasch Genootschap.

VKI : Verhandelingen van het Koninklijk Instituut.

(1) La colonie cam du Cambodge, qui est par contre restée liée au monde musulman par l'intermédiaire, ici encore, des Malais, pratiquait encore avant les évènements de ces derniêres un Islam plus orthodoxe. 


\section{BIBLIOGRAPHIE}

Al-Atras, S. Naguib

1969 Preliminary Statement on a General Theory of the Islamization of the Malay-Indonesian Archipelago. Kuala Lumpur.

Arnold, T.

1935 The Preaching of Islam. A History of the Propagation of the Muslim Faith. London.

Aymonier, E.

1889 "Grammaire de la langue chame», Excursions et Reconnaissances, XIV/31, 5-92.

1890 "Légendes historiques des Chams", Excursions et Reconnaissances, XIV/32, 145-206.

Ayres, Ch.

1904 Fernão Mendes Pinto. Subsidios para a sua biografia e para o estudo da sua obra. Lisboa.

BAROROH BARIED

1978 "Le Shi'isme en Indonésie ", Archipel, XV, 65-84.

Benedict, P. K.

1941 "A Cham Colony on the Island of Hainan ", Harvard Journal of Asiatic Studies, VI, 129-134.

Blair, H. et Robertson, J. A.

1903- The Philippine Islands. 1493-1898. Cleveland, 55 vols. 1909

Boisselier, J.

1963 La statuaire du Champa. (PEFEO LIV), Paris.

Boxer, G. R.

1969 "Portuguese and Spanish Projects for the Conquest of Southeast Asia ", Journal of Asian History, III/2, 118-136.

1970 "A Spanish Description of the Chams in 1595 ", in : Readings on Asian Topics, pp. 35-44 (Scandinavian Institute of Asian Studies, Monograph no 1), Lund.

Brandes, J. L. A.

1886 [Note sur la datation de l'inscription tombale de la Putri Cĕmpa], $N B G, \mathrm{XXIV,} \mathrm{p.} 42$. 
1900 Register op de Proza-omzetting van de Babad Tanah Jawi. ( $V B G \mathrm{LI} / 4)$, Batavia.

1920 Pararaton (Ken Arok) of Het Boek der Koningen van Tumapĕl en van Majapahit. (VBG LXII), Batavia.

BrigGs, L. P.

1949 "Spanish Intervention in Cambodia, 1593-1603 », T'oung-pao, XXXIX : 132-160.

1950 "Les missionnaires portugais et espagnols au Cambodge, 1555-1603 », BSEI, XXV/1 : 5-29.

Brown, G. C.

1952 "Sejarah Melayu or Malay Annals; a translation of Raffles Ms. 18 », JMBRAS, XXV/2-3:1-276.

Buch, W. J. M.

1936- "La Compagnie des Indes Néerlandaises et l'Indochine ", 1937 BEFEO, XXXVI : 97-196; XXXVII : 121-237.

Cabaton, A.

1904 «Une traduction interlinéaire malaise de la Aqīdah d'AlSenūsī », $J A$, III, 115-145.

1906 "Notes sur l'Islam dans l'Indochine française », $R M M$, I : 27-47.

1907 "Les Chams musulmans de l'Indochine française », $R M M$, II : 129-180.

1908 "Quelques documents espagnols et portugais sur l'Indochine aux $\mathrm{XVI}^{\mathrm{e}}$ et $\mathrm{XVII}^{\mathrm{e}}$ siècles ", $J A$, XII : 255-292.

1909 «Une intervention européenne au Cambodge à la fin du $\mathrm{XVI}^{\mathrm{e}}$ siècle ", Revue Indochinoise, XI : 1171-1188.

$1914 a$ Brève et véridique relation des événements du Cambodge par Gabriel Quiroga de San Antonio, Paris.

$1914 b$ "Les Hollandais au Cambodge au Xvire siècle", Revue de l'Histoire des Colonies françaises (tiré à part, Paris, 1914).

1914- "Le Mémorial de Pedro Sevil à Philippe III sur la conquête de

1916 l'Indochine (1603) ", Bulletin de la Commission archéologique de l'Indochine : 1-102.

1927 "L'Islam dans l'Indochine française", in : Encylopédie de l'Islam, Leiden, vol. II : 537-542.

Ch'en t'ieh-fan et Franke, W.

1973 "A Chinese Tomb Inscription of AD 1264, discovered recently in Brunei ", Brunei Museum Journal, III/1 : 91-99.

Ghiu Ling-Yeong

1967 "Sino-Javanese Relations in the Early Ming Period", in : Symposium on Historical, Archaeological and Linguistic Studies on Southern China, Southeast Asia and the Hong-kong Region, pp. 214-222. Hong-kong. 
Coolhaas, W. Ph.

1968 Generale Missiven (...) der Vereenigde Oostindische Compagnie, 's-Gravenhage, vol. III.

Cortesão, A.

1944 The Suma Oriental of Tomé Pires. (Hakluyt Society), London, 2 vols.

Cowan, H. K. J.

1933 "Het Atjèh'sch metrum "Sandja " in verband met en Tjamsch gedicht », $B K I, \mathrm{XG}, 149-155$.

1938 "Rectification de quelques affixes cams, rendus inexactement dans les grammaires ", Acta Orientalia, XVI, 181-191.

1939 [Compte rendu de Kern, 1938], Djåwå, XIX, 121-126.

1948 "Aanteekeningen betreffende de Verhouding van het Atjèhsch tot de Mon-Khmer-talen ", BKI, CIV, 429-514.

Damais, L.-Ch.

1957 "Les tombes musulmanes datées de Trålåyå ", BEFEO, XLVIII/2, 353-415.

1968 "L'épigraphie musulmane dans le sud-est asiatique », BEFEO, LIV, 567-604.

1970 Répertoire onomastique de l'épigraphie javanaise. (PEFEO, LXVI), Paris.

DAmes, M. L.

1918 The Book of Duarte Barbosa. (Hakluyt Society), London, 2 vols.

De Graaf, H. J. et Pigeaud, Th. G. Th.

1974 De eerste Moslimse vorstendommen op Java. (VKI 69), Leide.

DJAJADININGRAT, H.

1913 Critische Beschouwing van de Sadjarah Bantén. Haarlem.

Drewes, G. W. J.

1968 "New light on the Coming of Islam to Indonesia? ", BKI, CXXIV/4, 433-459.

Durand, E.-M.

$1903 a$ "Les Chams Bani", BEFEO, III, 54-62.

$1903 b$ "Le temple de Po Romé à Phan-rang », BEFEO, III, 597-603.

1905 "La Chronique royale », BEFEO, V, 377-382.

1907 "Le livre d'Anouchirvân », BEFEO, VII, 321-339.

FAtimi, S. Q.

1961 "The Role of China in the Spread of Islam in Southeast Asia ", in : First International Conference of Southeast Asian Historians, Singapore (ronéot.).

1963 Islam comes to Malaysia. Singapore.

Feng Chenguun 满永鈞

1935 Yingya shenglan jiaozhu 滰涯勝筧校注. Shanghai. 
Ferrand, G.

1913- Relations de voyages et textes géographiques arabes, persans et

1914 turks relatifs à l'Extrême-Orient. Paris, 2 vols.

1922 "L'empi re soumatranais de Çrīvijaya », JA, XX, 1-104.

Gerini, G. E.

1909 Researches on Ptolemy's Geography of Eastern Asia. London. Groslier, B. P. et Boxer, C. R.

1958 Angkor et le Cambodge au XVI siècle d'après les sources portugaises. Paris.

Hirth, F.

1896 Die Insel Hainan nach Chao Yu-kua. (Bastian Festschrift), Berlin.

Hirth, F. et Rockhill, W. W.

1911 Chau Ju-kua: His Work on the Chinese and Arab Trade in the twelfth and thirteenth centuries, entitled Chu-fan-chi. St-Petersburg.

Ho KÊE-ÊN 向格思

1959- “瑟族之确究. A Study on the Boat People of South Ghina》. $1960 \mathrm{Jl}$. of Oriental Studies, V, 1-40.

1967 "The Tanka or Boat People of South China ", in : Symposium on Historical, Archaeological and Linguistic Studies on Southern China, Southeast Asia and the Hong-kong region, pp. 120-123. Hong-kong.

HooykaAs, C.

1947 Over Maleise Literatur. Leiden.

JuYNBOLL, H. H.

1911 Supplement op den Catalogus van de Javaansche en Madoereesche handschriften der Leidsche Universiteits-Bibliotheek. Deel II: Nieuwjavaansche gedichten en oud-, middel-en nieuwjavaansche prozageschriften. Leiden.

KERN, R. A.

1938 "De verbreiding van den Islam ", in : F. W. Stapel, Geschiedenis van Nederlansdch-Indie ", vol. I, 305-365. Amsterdam.

KIKUCHI Saburō

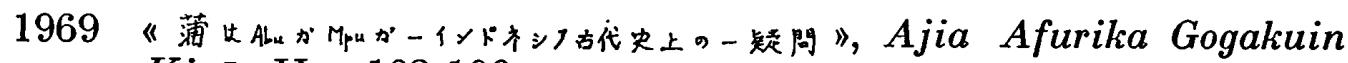
Kiy $\bar{o}$, II : 103-106.

Krom, N. J.

1931 Hindoe-Javaansche Geschiedenis. Den Haag..

Kuwabara Jitsuzō

1928- "On P'u Shou-keng, a man of the Western Regions, who was

1935 the Superintendant of the Trading Ships' Office in Ch'uan-chou towards the end of the Sung Dynasty ", Memoirs of the Research Department of the Toyō Bunko, II, 1-79; VII, 1-104. 
Lafont, P.-B., Po Dharma et Nara Vija

1977 Calalogue des manuscrits cam des bibliothèques françaises. (PEFEO, CXIV), Paris.

Launay, A.

1923 Histoire de la Mission de Cochinchine. Documents historiques, $I$ : 1658-1728. Paris.

Lo XIANG-LIN (Lo HsiANG-LIN) 羅香林

$1955 P u$ Shougeng zhuan 蒲素庚傳. Taibei.

$1959 \mathrm{Pu}$ Shougeng yanjiu 㜑尌庚研究. Hong-kong.

1967 "Islam in Canton in the Sung Period", in : Symposium on Historical, Archaeological and Linguistic Studies in Southern China, Southeast Asia and the Hong-kong region, pp. 177-179. Hong-kong.

LOMBARD, M.

1971 L'Islam dans sa première grandeur (VIII ${ }^{\mathrm{e}}-X I^{\mathrm{e}}$ siècle). Paris.

Mak Phoeun

1975 Les chroniques royales du Cambodge (de 1594 à 1677). Thèse de $3^{\mathrm{e}}$ cycle, Paris.

Manguin, P.-Y.

1972 Les Portugais sur les côtes du Việt-nam et du Campā. (PEFEO LXXXI), Paris.

1976 "La traversée de la Mer de Chine méridionale, des Détroits à Canton, jusqu'au xvir ${ }^{\mathrm{e}}$ siècle", in : Actes du $X X I X^{\mathrm{e}}$ Congrès International des Orientalistes (Paris 1973). Section Asie du Sud-Est continentale, vol. 2, 110-115. Paris.

Marre, A.

1895 "Madiapahit et le Tchampa ", in : Publication du Centenaire de l'École des Langues Orientales, pp. 93-113. Paris.

Marrison, G. E.

$1951 a$ "The Chams of Malacca", JMBRAS, XXIV/1, 90-98.

$1951 b$ "The Coming of Islam to the East Indies", JMBRAS, XXIV/1, 28-37.

Maspero, G.

1928 Le royaume de Champa. Paris.

MAYERS, W. F.

1874- "Chinese Explorations of the Indian Ocean during the fifteenth century ", The China Review, III/7, 219-225; III/6, 320-331.

Mills, J. V. G.

1970 Ying-yai sheng-lan. The Overall Survey of the Ocean Shores. (Hakluyt Society), Cambridge.

Miguel, A.

1975 La géographie humaine du monde musulman. Vol. II, Paris. 
Mori Katsumi

1972 "The Beginning of Overseas Advance of Japanese Merchant Ships ", Acta Asiatica, XXIII, 1-24.

Moulle, A. G. et Pelliot, P.

1938 Marco Polo. The Description of the World. London, 2 vols.

Moura, J.

1883 Le Royaume du Cambodge. Paris, 2 vols.

Mus, P.

1931 "Religion des Chams ", in : S. Lévi (éd.), L'Indochine, vol. I, 144-156. Paris.

NER, M.

1941 "Les Musulmans de l'Indochine française", $B E F E O$, XLI, 151-200.

NoORDUYN, J.

1956 "De Islamisering van Makasar », BKI, CXII, 247-266.

Olthof, W. L.

1941 Babad Tahah Jawi in proza (...) naar de Uitgave van $J . J$. Meinsma 's-Gravenhage.

Pelliot, P.

1963 Notes on Marco Polo. Paris, vol. II.

PÉri, N.

1923 «Essai sur les relations du Japon et de l'Indochine aux xvi et $\mathrm{XVII}^{\mathrm{e}}$ siècles ", BEFEO, XXIII, 1-136.

Phillips, G.

1879- "Nestorians at Canton", China Review, VIII, 31-34. 1880

Piat, M.

1974 "Les Chroniques royales khmer», BSEI, XLIX/1, 35-144.

Pigeaud, Th. G.

1967- Literature of Java. The Hague, 3 vols. 1970

Po Dharma

1978 Chroniques du Pānduranga. Introduction, textes et traductions annotées. Diplôme de l'École Pratique des Hautes Études, IVe section, Paris.

RAs, J. J.

1968 Hikayat Bandjar. A Study in Malay Historiography. (Bibliotheca Indonesica, I), The Hague.

Ravaisse, P.

1922 "Deux inscriptions coufiques du Campa », $J A, \mathrm{XX}, 247-289$.

1925 "L'inscription coufique de Léran à Java », $T B G, \mathrm{LXV}$, 668-703. 


\section{ReINAUD}

1848 Géographie d'Aboulféda. Tome 1 : Introduction générale à la géographie des Orientaux. Paris.

Ribeiko, L.

1961 «Uma geografia quinhentista », Studia, VII, 151-318.

RigG, J.

1851 "Ancient Javanese Inscriptions at Panataram ", Journal of the Indian Archipelago and Eastern Asia, V, 439-440.

SAUVAGET, J.

1948 'Ahbār as-sin wa l-hind. Relation de la Chine el de l'Inde rédigée en 851. Paris.

Savina, M.

1929 Monographie de Hainan. Hanoi.

SCHRIEKE, B.

1957 "Ruler and Realm in Early Java », in : Indonesian Sociological Studies. vol. II, 1-268. The Hague.

Shellabear, W, G.

1910 Sejarah Malayu or the Malay Annals. (Malay Literature Series, IX), Singapore, 2 vols.

1914 Hikayat Hang Tuah. (Malay Literature Series, III), Singapore, 4 vols.

ShorTo, H. L.

1975 "Achinese and Mainland Austronesian》, Bulletin of the School of Oriental and African Studies, XXXVIII/1, 81-102.

SNouck Hurgronje, C.

1906 The Achehnese. 2 vols. Leyden.

STEIN. R.

1947 "Le Lin-yi, sa localisation, sa contribution à la formation du Champa et ses liens avec la Chine ", Han Hiue, II/1-3.

STÜBEL, H.

1937 Die Li-stämme der Insel Hainan. Berlin.

TASAKa Kōdō

1952 《占城日教史序説》, Tōhōgaku, IV, 52-60.

TeEuw, A. et Wyatt, D. K.

1970 Hikayat Patani. The Story of Patani. (Bibliotheca Indonesica, V), The Hague, 2 vols.

TibBetts, G. R.

1957 "Early Muslim Traders in South-East Asia ", JMBRAS, $\mathrm{XXX} / 1,1-45$.

VAN DiJk, L. C. D.

1862 Neêrland's vroegste betrekkingen met Borneo, den Solo-archipel, Cambodja, Siam en Cochinchine. Amsterdam. 
Vreede, A. C.

1892 Catalogus van de Javaansche en Madoereesche handschriften der Leidsche Universiteits-Bibliotheek. Leiden.

Winstedt, R. O.

1917 "The Advent of Muhammadanism in the Malay Peninsula and Archipelago ", JSBRAS, LXXVII, 171-175.

1938 "The Malay Annals, or Sejarah Melayu ", JMBRAS, XVI/3, $1-226$.

XIANG Da 向连 (Éd.)

1961 Xiang fanguo zhi 西洋番团志. Pekin.

Yамамото Tatsuro

1936 "On Tawālisī described by Ibn Baṭūta », Memoirs of the Research Department of the Tōyo Bunko, VIII, 93-133.

Yule, H. et Cordier, H.

1913- Cathay and the Way thither. (Hakluyt Society), London, 19164 vols. 